\title{
PBAF regulates compartmentalization of actively transcribing chromatin hubs
}

Charles A. Kenworthy ${ }^{1}$, Shu-Hao Liou ${ }^{1}$, Panagiotos Chandris ${ }^{2}$, Vincent Wong ${ }^{1}$, Patrycja Dziuba $^{1}$, Luke D. Lavis ${ }^{3}$, Wei-Li Liu ${ }^{1}$, Robert H. Singer ${ }^{1,3}$, and Robert A. Coleman ${ }^{1 *}$

${ }^{1}$ Gruss-Lipper Biophotonics Center, Department of Anatomy and Structural Biology, Albert Einstein College of Medicine, Bronx, New York 10461, USA

${ }^{2}$ Section on High Resolution Optical Imaging, National Institute on Biomedical Imaging and Bioengineering, National Institutes of Health, Bethesda, Maryland, 20892, USA

${ }^{3}$ Janelia Research Campus, Howard Hughes Medical Institute, Ashburn, Virginia, 20147

*To whom correspondence should be addressed.

Gruss-Lipper Biophotonics Center, Department of Anatomy and Structural Biology, Albert Einstein College of Medicine, Bronx, NY 10461 Tel: 718-430-8623; Email: robert.coleman2@einstein.yu.edu

Running Title: PBAF's dynamic chromatin targeting for genome organization

Keywords: PBAF, chromatin remodeling, Histone acetylation, Histone H3.3, Single Molecule Tracking, bromodomain, nuclear compartments, hubs 


\section{Abstract}

42

43 Transcriptionally active genes contain acetyl-rich chromatin and are organized in distinct

44 nuclear compartments that are spatially separated from transcriptionally inactive genes. It

45 is not known how this compartmentalized acetylated chromatin is targeted and regulated

46 by chromatin remodelers such as PBAF. Thus, we sought to understand how PBAF

47 targets chromatin and modulates compartmentalization of transcriptionally active genes

48 using live-cell single molecule fluorescence microscopy. Our work reveals chromatin

49 hubs throughout the nucleus where PBAF cycles on and off the genome. Deletion of

50 PBAF's bromodomains impairs recognition of hubs and cycling on chromatin.

51 Interestingly, markers for transcriptionally active and inactive genes can be found in

52 compartments harboring acetylated chromatin at the periphery that is selectively

53 recognized by PBAF via bromodomains. Defects in PBAF's peripheral targeting lead to a

54 select reduction in the size and number of compartments containing transcriptionally

55 active genes. Our data, combined with previous work in Yeast and Drosophila, suggest

56 that PBAF activity serves as a barrier to heterochromatin spreading. Overall, our findings

57 suggest that chromatin compartments are highly structured with unique peripherally

58 associated acetylation marks. PBAF utilizes these marks to help shape nuclear

59 compartments containing transcriptionally active genes, thereby aiding genomic

60 organization. 


\section{Introduction}

Hi-C studies have shown that genomic regions spanning up to a megabase, contain transcriptionally active genes rich in acetylated chromatin that are grouped together in "A" compartments ${ }^{1,2}$. Neighboring "B" compartments contain inactive chromatin with repressive epigenetic marks and are segmented away from A compartments ${ }^{2}$. High resolution Hi-C maps further identified a second level of local genome organization consisting of topologically associated domains (TADs) spanning sub-megabase regions and loop domains. High-resolution imaging using DNA-FISH in fixed cells has also spatially identified distinct active and inactive compartments that corresponded with genomic studies ${ }^{3,4}$.

A number of recent genomic studies have linked dynamic changes in genome topology with chromatin states and gene regulation during cellular stress, cell reprogramming and viral infection ${ }^{5-7}$. Specifically, transcription factor binding and expression of stress response and developmentally regulated genes are often linked to $\mathrm{A} / \mathrm{B}$ compartment switching at the end of these genes and boundaries between select $\mathrm{TADs}^{5-7}$. However, it is unclear how transcription factors dynamically recognize and alter these $\mathrm{A} / \mathrm{B}$ compartment boundaries to regulate genome topology at the single cell level.

PBAF, an ATP dependent chromatin remodeling complex, regulates transcription of stress response genes in Humans, C. elegans and Yeast via bromodomain dependent targeting of acetylated chromatin at promoters ${ }^{8-10}$. The yeast homolog of PBAF, RSC, has also been shown to alter genome topology likely via modulation of chromatin state and

84 transcription at genes within the boundaries between chromatin domains ${ }^{11}$. Therefore 85 understanding how PBAF dynamically recognizes different chromatin states in genomic 
86 compartments in vivo may lead to a greater understanding of the spatial and dynamic

87 regulation of transcription and genome topology.

88 Recent live-cell imaging studies indicate that a number of transcription factors (e.g.

89 RNA Polymerase II, Mediator and Sox2) dynamically bind chromatin as clusters to form

90 hubs that regulate local gene expression ${ }^{12-17}$. However, such dynamic activity of

91 transcriptional regulators, including chromatin modifiers, within distinct chromatin

92 compartments is currently poorly characterized due to a number of technical limitations.

93 In particular, researchers lack efficient methods to identify and quantitatively characterize

94 chromatin in active/inactive compartments in live cells.

95 To spatially distinguish and characterize different chromatin compartments in

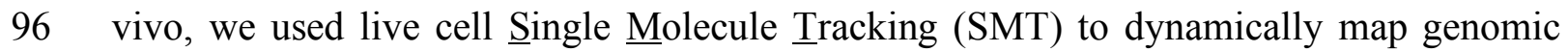

97 binding of PBAF alongside markers of transcriptionally active (H3.3) and inactive (HP1 $\alpha)$

98 genes. Our dynamic imaging studies reveal small hubs where PBAF cycles on and off

99 chromatin, which can be further grouped to form large compartments. We next sought to

100 address the pivotal question of how distinct compartmentalized chromatin states are

101 targeted by PBAF. Thus, we investigated PBAF's spatial distribution, velocities and

102 stability on chromatin when encountering H3.3 and HP1 $\alpha$ marked compartments. More

103 importantly, we have assessed the role of PBAF's bromodomains in hub targeting,

104 cycling on chromatin, select compartment engagement and genomic organization.

105 Overall, our studies provide new insights into dynamic chromatin targeting of PBAF via

106 bromodomains, which regulates compartmentalization of transcriptionally active genes to

107 aid in genomic organization. 


\section{Results}

\section{PBAF targets chromatin in distinct nuclear hubs and compartments}

111 Active genomic compartments are enriched in acetylated chromatin and

112 transcriptionally active genes ${ }^{1,18}$. PBAF targets a variety of acetylated residues, including

$113 \mathrm{H} 3 \mathrm{~K} 14 \mathrm{ac}$ and $\mathrm{H} 3 \mathrm{~K} 27 \mathrm{ac}$, on chromatin via its 8-12 bromodomains based on in vitro

114 studies $^{19,20}$. Therefore, we hypothesized that PBAF would dynamically localize to

115 discrete nuclear compartments that were enriched in acetylated chromatin in vivo (Figure 116 1a).

117 To characterize the dynamic binding of PBAF to chromatin in vivo, we chose to 118 fluorescently tag the BAF180 subunit (i.e. Halo-BAF180 WT, Figure S1), since it harbors

119 six bromodomains critical for interaction with acetyl-lysine residues on histones ${ }^{21,22}$.

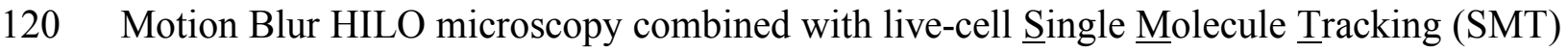

$121{ }^{23}$ was used to dynamically detect PBAF molecules stably bound to chromatin (Figure $1 \mathrm{~b}$

122 and Supplemental Movie S1). At long camera exposure rates ( 500 ms), fast-diffusing

123 nuclear PBAF complexes are blurred out and cannot be localized. Single PBAF

124 molecules, stably bound to chromatin targets, appear as distinct Point $\underline{\text { Spread }}$ Functions

125 (PSFs) that can be spatially and temporally resolved (Figure 1b). PSFs, representing

126 PBAF's binding and unbinding on chromatin, appear and disappear stochastically

127 throughout the time course of imaging (Figure $1 \mathrm{~b}$ and Supplemental Movie S1). We

128 confirmed that these Halo-tagged BAF180 proteins were incorporated into the large

129 multi-subunit PBAF complex via co-immunoprecipitation studies and live-cell fast

130 diffusion measurements (Figures S1 and S2). 
131 Two dimensional (2D) projection maps showed select nuclear regions that contained

132 high densities of PBAF's chromatin binding events alongside deserts devoid of binding

133 (Figures 1c, left panel, and S3c). Based upon these maps, we hypothesized that PBAF's

134 distinct localization pattern was due, in part, to dynamic interactions between PBAF's

135 bromodomains and acetylated chromatin. Therefore, we developed an approach to

136 spatially define the frequency of PBAF's chromatin binding within nuclear subregions.

137 These dynamic binding frequency heat maps were generated by raster scanning across the

138 nucleus and counting the number of PBAF-chromatin binding events in a given sized

139 window (binding events $/ \mu \mathrm{m}^{2} / \mathrm{sec}$ ) (Figures 1c and 1d). Spatially isolated regions

140 representing high frequency PBAF binding to chromatin were scattered throughout the

141 nucleus (Figure 1d, yellow spanning to red regions).

142 Analysis with the smallest scanning window $\left(0.064 \mu \mathrm{m}^{2}\right)$ revealed hubs of repeated

143 PBAF binding events to target loci within an area of $\sim 250 \mathrm{~nm}$ in diameter (Figure 1d, top

144 panel). Importantly, no hubs were detected in simulations where localizations of an

145 equivalent number of binding events were randomized throughout the nucleus (Figure

146 S4a). These results are consistent with previous studies showing hubs of EWS/FLI1,

147 Sox2 and RNA Polymerase II at enhancers and promoters in nuclear domains of roughly

148 equivalent sizes $^{13,14,16,24}$. Therefore, we speculate that these PBAF hubs represent

149 repeated binding or "cycling" on nucleosomes in small genomic regions at enhancers and

150 promoters.

151 The largest scanning window $\left(2.54 \mu \mathrm{m}^{2}\right)$ indicated clusters of binding hubs grouped

152 together to form a large compartment $(\sim 1.7 \mu \mathrm{m}$ in diameter, Figure 1d, right panel).

153 Importantly, the average size of our PBAF binding compartments in live cells was only 
154 slightly larger than active and inactive chromatin compartments measured by super-

155 resolution microscopy in fixed cells ${ }^{4}$. Overall, these results suggest that PBAF is likely

156 targeting chromatin in a permissive state over select genomic regions that are packaged in

157 confined hubs that are grouped in compartments.

158 PBAF targeting and cycling on chromatin hubs is regulated by BAF180

\section{9 bromodomains}

160 It is generally thought that arrays of acetylated nucleosomes are repeatedly targeted

161 by bromodomain containing chromatin remodeling complexes such as PBAF (Figure

162 2a $)^{25}$. Multiple PBAF subunits contain bromodomains that recognize a large variety of

163 acetyl-lysine residues in chromatin ${ }^{20,26-28}$. Notably, BAF180 contains six bromodomains

164 while BRG1 and BRD7 each contain one bromodomain. Therefore, we hypothesized that

165 BAF180 bromodomains would have major role in PBAF's targeting and cycling on hubs

166 (Figure 2a).

167 To determine if chromatin hubs were targeted via PBAF bromodomain/acetyl-lysine

168 interactions, we compared the high frequency binding of wild-type PBAF and a mutant

169 PBAF lacking the six BAF180 bromodomains (i.e. $\triangle \mathrm{BD}$ ) (Figure 2b). Interestingly, the

170 number of PBAF binding hubs in each cell was reduced upon deletion of BAF180

171 bromodomains (Figure 2c). Correspondingly, the number of PBAF binding hubs

172 increased in a BAF180 bromodomain-dependent manner when global levels of histone

173 acetylation were elevated via SAHA treatment (Figures 2c and S4b). Overall, our data

174 indicate that target hubs are defined by repeated rounds of PBAF binding to chromatin

175 via BAF180 bromodomains/acetyl-lysine interactions. 
176 We next investigated how chromatin in target hubs is temporally occupied by PBAF.

177 Chromatin hubs are occupied via a series of PBAF binding and unbinding events

178 interspersed with latent periods of non-occupancy (Figure 2d). We found that both wild-

179 type PBAF and mutant PBAF $\triangle B D$, on average, exhibited $\sim 9-10$ rounds of repeated

180 binding at target hubs over $\sim 18$ minutes of continuous imaging (Figure 2e). Furthermore,

181 elevated histone acetylation levels led to a slight but significant increase in the number of

182 PBAF binding cycles at target hubs for both wild-type and mutant PBAF (Figure 2e). We

183 speculate that residual bromodomains in mutant PBAF's BRD7 and BRG1 subunits may

184 compensate to increase cycling on select arrays of hyperacetylated nucleosomes.

185 To assess further assess the molecular origins of cycling on chromatin hubs,

186 histograms of PBAF latency times were generated and fit to single, double and triple 187 exponential decay models (Figure S4b). Statistical analysis indicated that a triple 188 exponential decay model best fit the distribution of PBAF latency times. This enabled us 189 to classify latency times as either short $(\sim 4.5 \mathrm{sec})$, intermediate $(\sim 21 \mathrm{sec})$ or long $(\sim 205$ 190 sec) (Figure S4b). Removal of BAF180 bromodomains altered PBAF's latency at hubs

191 (Figures 2f and S4d), specifically increasing the long latency time of mutant PBAF

192 cycling events (compare WT vs $\Delta \mathrm{BD}$ ). Notably, this difference in latency between wild-

193 type and mutant PBAF was also further enhanced on hyperacetylated chromatin (Figure 194 2f, SAHA treatment). Therefore, PBAF's cycling and re-occupancy of acetylated 195 chromatin hubs may be aided via BAF180 bromodomains.

\section{Dynamic mapping of PBAF, transcriptionally active and heterochromatic}

\section{7 compartments}


198 Thus far, our dynamic binding frequency heat maps allow us to quantitate PBAF

199 target hubs grouped in close proximity (Figure 1d). We have termed these grouped hubs

200 as PBAF binding compartments given their large size and distinct localization patterns

201 (Figures 1d and 3a). Previous studies have established that PBAF binds nucleosomes at

202 promoters of both transcriptionally active and repressed genes ${ }^{19,29-32}$. To test if PBAF

203 localizes on chromatin in different compartment types, we used a similar strategy as

204 above to dynamically image transcriptionally active (H3.3-SNAP) and heterochromatic

205 (SNAP-HP1 $\alpha$ ) nuclear subregions in single cells (Supplemental Movies S2 and S3).

206 Compartments containing dense high frequency H3.3-SNAP genomic interactions were

207 found within distinct subregions in the nucleus (Figure 3b, left panel). Likewise,

208 heterochromatic compartments harboring high frequency SNAP-HP1a/chromatin

209 interactions were also observed (Figure 3b, right panel). HP1 $\alpha$ compartments defined in

210 this manner roughly resembled the size of phase-separated heterochromatin ${ }^{33}$. These

211 results demonstrate that we can identify large transcriptionally active and inactive regions

212 of the genome clustered in select compartments in a live cell.

213 Our analysis allows us to quantitate the number and sizes of compartments in a

214 single cell. Interestingly, the number of PBAF compartments was reduced with a minimal

215 impact on their size in our mutant PBAF $\triangle \mathrm{BD}$ expressing cells (Supplemental Figures

216 S5a and S5b). These results suggest that a subset of PBAF compartments is defined via

217 BAF180 bromodomain targeting to select acetylation marks in chromatin hubs. We also

218 found that the number and size of the H3.3 compartments were significantly diminished

219 in our mutant PBAF BAF180 $\triangle \mathrm{BD}$ cells (Figures 3c and 3d). Therefore, PBAF may be

220 regulating the establishment of a subset of $\mathrm{H} 3.3$ compartments. Moreover, there were no 
221 differences in the number and size of HP1 $\alpha$ compartments between wild-type and mutant

222 PBAF cells (Figures 3c and 3d). Overall, our results suggest that establishment of H3.3

223 compartments, in part, requires PBAF's BAF180 bromodomains.

224 Select spatial targeting of chromatin in transcriptionally active and heterochromatic

225 compartments

226 To further reveal any spatial correlations between different compartments, dual color

227 single molecule imaging was performed to map PBAF's binding to chromatin alongside

228 transcriptionally active or inactive compartments inside a single cell (Figure 4). First, we

229 observed some high frequency PBAF binding that overlapped with both transcriptionally

230 active and heterochromatic compartments (Figure 4a). In terms of total compartment area,

231 approximately $27 \%$ and $24 \%$ of our mapped PBAF compartments overlapped

232 respectively with the H3.3 and HP1 $\alpha$ compartments (Figure 4b). Collectively, over 50\%

233 of the total area of PBAF's compartments coincide with compartments containing H3.3

234 and HP1 $\alpha$ marked chromatin. We surmise that the chromatin within the remaining non-

235 overlapping regions of PBAF compartments might be associated with additional subtypes

236 of inactive compartments not marked by HP1 $\alpha^{2}$.

237 Next, to better characterize chromatin states within compartments, PBAF SMT

238 trajectories within H3.3 or HP1 $\alpha$ compartments were isolated (Figures S6a and S6b).

239 Roughly $23 \%$ and $21 \%$ of PBAF's total nuclear chromatin binding events respectively

240 occurred within transcriptionally active H3.3 or HP1 $\alpha$ marked compartments (Figure 4c).

241 Notably, upon close inspection, a large number of PBAF's chromatin binding events

242 localized to the periphery of H3.3 or HP1 $\alpha$ marked compartments (Figure 4d). 
To quantitatively determine PBAF's preferential targeting inside compartments, we

244 examined the ratio between the number of PBAF binding events inside of compartments

245 (nPBIC) and the area of compartments (nAC, see methods). A value greater than 1 for the

$246 \mathrm{nPBIC} / \mathrm{nAC}$ ratio indicates enrichment of PBAF binding inside a specific type of

247 compartment relative to global chromatin. A value less than 1 for the $\mathrm{nPBIC} / \mathrm{nAC}$ ratio

248 indicates depletion of PBAF binding inside compartments. Overall, we observed an

249 enrichment of PBAF binding inside the H3.3 and HP1 $\alpha$ compartments (i.e. Entire Comp.

250 shown in Figure 4e, top panels).

251 To pinpoint where PBAF was mostly enriched inside compartments, we then plotted

$252 \mathrm{nPBIC} / \mathrm{nAC}$ ratios as a function of PBAF's distance away from the compartment

253 periphery (Figure 4e). We found a significant differential enrichment of wild-type

254 PBAF's chromatin binding within $\sim 84 \mathrm{~nm}$ of the periphery inside of H3.3 and HP1 $\alpha$

255 compartments (top panels). In stark contrast, mutant PBAF that lacks BAF180

256 bromodomains (i.e. $\Delta \mathrm{BD}$ ) displayed no spatial enrichment close to the periphery of $\mathrm{H} 3.3$

257 and HP1 $\alpha$ compartments (bottom panels). Thus, these findings reveal that chromatin at

258 the edge of both transcriptionally active and heterochromatic compartments likely

259 contains distinct acetylation marks that target PBAF via BAF180 bromodomains.

260 To support our live-cell dynamic tracking of PBAF binding on select distinct

261 chromatin, super-resolution STORM microscopy in fixed cells was also conducted

262 (Figure S7). The analysis validates the overlap between wild-type PBAF and H3.3/HP1 $\alpha$

263 marked chromatin (Figure S7, cyan). In many subnuclear regions, PBAF binds chromatin

264 and forms a distinct close interface surrounding H3.3 marked chromatin domains (Figure

265 S7, bottom left panel, highlighted in red stars), consistent with the results in Figure 4. A 
266 similar but less pronounced pattern was seen with PBAF and HP1 $\alpha$ marked chromatin

267 domains (Figure S7, bottom right panel). Taken together, these studies illustrate that

268 PBAF can selectively bind distinct transcription-regulated chromatin via BAF180's

269 bromodomains.

270 Transcriptionally active versus heterochromatic chromatin is differentially engaged

271 by PBAF

272 Chromatin states are thought to significantly differ between transcriptionally active

273 and heterochromatic compartments ${ }^{1,18}$. To further characterize these different chromatin

274 states, we determined PBAF's genomic residence time in transcriptionally active H3.3

275 and heterochromatic HP1 $\alpha$ compartments (Figure 5a). Residence time analysis revealed

276 two chromatin-binding populations of PBAF in a representative H3.3 compartment

277 (Figure 5b, red line). The predominant PBAF population ( $\sim 83 \%$ of molecules) transiently

278 bound chromatin for $\sim 0.8$ seconds. This PBAF population most likely represents non-

279 specific binding since residence time analysis for the Halo-tag alone also showed brief

$280(0.8 \mathrm{sec})$ interactions with chromatin ${ }^{23}$. The remaining PBAF molecules $(\sim 17 \%)$ stably

281 bound to chromatin in this H3.3 compartment for $\sim 9$ seconds on average. Importantly, we

282 are measuring PBAF's dissociation from chromatin, as its residence time $(9 \mathrm{sec})$ is

283 significantly shorter than our dye photobleaching rates ( $\mathrm{t}_{1 / 2}$ of approximately 100-200

$284 \mathrm{sec})$.

285 PBAF's residence time broadly varied from $\sim 7-22$ seconds within different H3.3

286 compartments in the same cell (Figure 5c). Global analysis reveals two subclasses of

287 H3.3 compartmentalized chromatin across different cells that are targeted by PBAF

288 (Figure 5d, green line). PBAF bound to chromatin for $\sim 8$ seconds in the majority of H3.3 
compartments, with a minor population of compartments exhibiting a residence time of

29016 seconds. Comparatively, there was only one class of heterochromatic HP1 $\alpha$

291 compartments displaying an average PBAF residence time of $\sim 12$ seconds on chromatin

292 (Figure 5d, red line). Overall, the majority of PBAF molecules that localized to H3.3

293 marked chromatin bound for a shorter duration than tracks within HP1 $\alpha$ regions (Figure

294 5d). These results indicate that our dynamic imaging can segment compartments

295 containing different chromatin states. Furthermore, this underlying chromatin state of

296 target nucleosomes in different compartments affects PBAF's binding.

297 To assess if BAF180 bromodomains regulate PBAF's stability on chromatin, we 298 examined the residence time of mutant PBAF $\triangle \mathrm{BD}$ on chromatin globally (Figure S8)

299 and in compartments (Figure 5e). Mutant PBAF $\triangle \mathrm{BD}$ had a decreased percentage of

300 stable global chromatin binding events, indicating an inefficient target search mechanism

301 (Figure S8a). Compared to wild-type PBAF, mutant PBAF $\triangle B D$ had reduced residence

302 times on chromatin globally (Figure S8b) and in high frequency PBAF compartments

303 (Figure S8c). Thus, PBAF lacking BAF180 bromodomains has defects in associating

304 with and anchoring to chromatin. Intriguingly, while there was a significant reduction in

305 stability on global chromatin, mutant PBAF $\triangle \mathrm{BD}$ exerted little difference in residence

306 times between transcriptionally active H3.3 and heterochromatic HP1 $\alpha$ compartments

307 (Figure 5e). Notably, PBAF $\triangle \mathrm{BD}$ exhibited a bimodal distribution profile of residence

308 times $(\sim 8$ and $\sim 13 \mathrm{sec})$ within heterochromatic HP1 $\alpha$ compartments. Therefore, a

309 subclass of distinct heterochromatic compartments is defective for interacting with PBAF

$310 \Delta \mathrm{BD}(\sim 8 \mathrm{sec})$ compared to PBAF WT (residence time $\sim 12 \mathrm{sec}$, Figure $5 \mathrm{~d}$ versus $5 \mathrm{e})$. We

311 speculate that PBAF may target at least two different types (i.e. BAF180 bromodomain 
312 dependent and independent) of HP1 $\alpha$-marked heterochromatic compartments via distinct

313 subunits and mechanisms.

\section{Surveying the local chromatin environment of PBAF target loci}

317 nucleosomes along the $\mathrm{DNA}^{34,35}$. In the cell, PBAF's movement when bound to a

318 genomic scaffold could be due to (1) translocation along the DNA, (2) local constrained

319 diffusion of the genomic DNA that is dependent on local chromatin density, or (3) a

320 combination of both translocation and DNA diffusion. Regardless of its origin, we

321 surmised that PBAF's movement could serve as a probe of the local condensation status

322 of chromatin (Figure 6a). Thus, we hypothesized that PBAF would move faster when

323 bound to nucleosomes within open euchromatic versus condensed heterochromatic loci

324 (Figure 6a).

325 To study PBAF's movement on chromatin, we measured the frame-to-frame X, Y

326 positional displacements of individual genome bound PBAF SMT trajectories (Figure 6b).

327 Interestingly, both unfiltered (thin blue/yellow lines) and smoothed (thick dark blue and

328 orange lines) SMT trajectories of chromatin bound PBAF predominantly displayed

329 asymmetric elongated curvilinear movement (Figure 6b). This movement was not due to

330 cell migration or microscope drift as trajectories occurring in close spatial and temporal

331 proximity often moved in opposing or distinct directions. Our results were consistent

332 with previous studies showing that chromatin moves via a curvilinear mechanism ${ }^{36,37}$.

333 We next sought to address whether different chromatin states of target

334 nucleosomes would impact PBAF's velocity. To begin tackling this task, we

335 quantitatively characterized the movement of individual trajectories (Figure S9a). The 
336 velocity of PBAF's curvilinear movement was discontinuous, occurring in a series of

337 starts and stops. Furthermore, multiple Gaussian model fitting of the individual

338 trajectory's average velocities revealed at least three to four distinct populations of PBAF

339 velocities (Figure 6c). These four populations may reflect four different chromatin states

340 bound by PBAF. For clarity, we combined the two fast PBAF movement populations into

341 a single category. We suspected that less crowded euchromatic loci would allow fast

342 movement of PBAF (Figure 6a, left panel). Correspondingly, heterochromatin would

343 result in medium and slow moving PBAF (Figure 6a, right panel). Notably, throughout

344 the nucleus, approximately 77\% of PBAF's global chromatin binding events displayed

345 medium and slow velocities on chromatin (Figure 6c). This suggests that the majority of

346 chromatin throughout the nucleus is in a densely packed heterochromatic state (Figure 6a,

347 right panel).

348 Next, we compared PBAF's movement when bound to H3.3 versus HP1 $\alpha$ marked

349 chromatin (Figure 6d). HP1 $\alpha$ marked chromatin compartments contain a significantly

350 higher percentage of slow moving PBAF relative to H3.3 containing chromatin.

351 Correspondingly, open euchromatic H3.3 marked chromatin harbors an elevated

352 percentage of fast moving PBAF molecules versus condensed heterochromatic loci

353 (Figure 6d), likely reflecting differences in the local nucleosome density surrounding 354 target loci.

355 Throughout the nucleus, global chromatin is relatively closed and thought to 356 contain predominantly the histone $\mathrm{H} 3.1$ variant in place of $\mathrm{H} 3.3^{38}$. Therefore, as a control,

357 we further examined PBAF's velocity outside of H3.3 marked compartments. Indeed, 358 there was an increased percentage of slow moving wild-type PBAF bound to H3.1 
359 marked chromatin outside of H3.3 marked compartments (i.e. 37\% vs. 21\%, Figures S9b

360 and 6d). On the other hand, we observed a decrease in the slow moving PBAF population

361 outside of HP1 $\alpha$ marked compartments (i.e. $35 \%$ vs. 42\%, Figure S9b and $6 \mathrm{~d}$ ). This

362 suggests that the global chromatin on average is slightly less densely packed compared to

363 HP1 $\alpha$ marked chromatin. Collectively, PBAF's differential velocities likely reflect

364 chromatin compaction, which increases from H3.3 to H3.1 to HP1 $\alpha$ marked chromatin

365 states. However, we can't exclusively rule out the possibility that PBAF may bind these

366 distinct types of chromatin via different mechanisms and subunits, resulting in distinct

367 velocities regardless of compaction states.

368 Residence time analysis indicated that removal of PBAF's bromodomains altered

369 binding in heterochromatic compartments (Figure 5e). Therefore, we exploited mutant

370 PBAF $\triangle B$ D's velocity to infer the local chromatin density of its targets within

371 heterochromatin. Interestingly, we observed a decreased percentage of slow moving

372 mutant PBAF $\triangle \mathrm{BD}$ molecules relative to wild-type PBAF in heterochromatic HP1 $\alpha$

373 compartments (Figure 6d). It is important to note that these results are consistent with the

374 residence time analysis (Figures $5 \mathrm{~d}$ and 5e), implying mistargeting of mutant PBAF $\triangle \mathrm{BD}$

375 to potentially unstable nucleosomes within heterochromatic compartments. Taken

376 together, our studies suggest that PBAF uses bromodomains for targeting distinct

377 chromatin states within heterochromatic compartments. 
Discussion

A battery of work has now established that transcriptionally co-regulated genes

381 are clustered in compartments containing Topologically Associated Domains (TADs) or

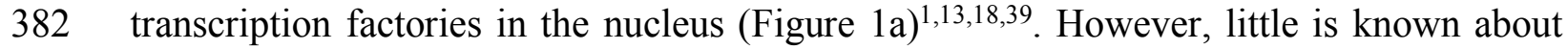

383 how these compartments are dynamically targeted and shaped by chromatin modifiers.

384 We proposed that PBAF could act to remodel chromatin in promoters, enhancers and 385 boundary elements in distinct subnuclear compartments. Using dynamic live-cell single

386 molecule tracking, chromatin binding frequency heat maps allowed us to visualize

387 compartmentalized chromatin binding of PBAF at different scales dependent on the size

388 of our scanning window (Figure 1d). As expected, different chromatin interacting

389 proteins, such as the H3.3 histone variant and the HP1 $\alpha$ heterochromatic binding protein,

390 also displayed compartmentalized binding in our live-cell imaging analysis (Figure $3 b$ ).

391 Therefore, we and other research groups have established that dynamic single molecule

392 tracking is useful for identifying and characterizing compartmentalized genomic binding

393 of a broad range of nuclear factors ${ }^{16,17}$.

394 Large chromatin domains $(\sim 2-30 \mathrm{~kb})$ can be found to be acetylated throughout the

395 genome 40,41 . We proposed that the discrete PBAF hubs identified in our heat maps

396 (Figure 1d) represent repeated binding to arrays of closely spaced acetylated nucleosomes

397 during our live-cell imaging (Figure 2). Indeed, removal of BAF180's bromodomains

398 reduced the number of PBAF hubs, while elevated global histone acetylation increased

399 the number of hubs (Figure 2c). Therefore, we surmise that PBAF binding hubs represent

400 acetylated loci (Figure 7a). Interestingly, our latency analysis reveals that BAF180

401 bromodomains also increase PBAF cycling on acetylated chromatin in hubs (Figure 2f). 
402 Therefore, our studies support that PBAF utilizes bromodomain/acetyl interactions to

403 remain in the immediate vicinity of target chromatin, likely facilitating repeated rounds

404 of remodeling events on nucleosomal arrays (Figures $2 \mathrm{a}$ and $7 \mathrm{a}$ ).

405 Our data visualized large compartments encompassing grouped hubs of 406 transcriptionally active genomic regions (Figure 3b, left panel). This result complements

407 Hi-C genomic studies ${ }^{1,18}$, indicating that compartmentalized regions of the genome 408 contain acetylated histone marks associated with active transcription. Notably, formation 409 of transcriptionally active compartments is likely regulated via PBAF's peripheral 410 targeting of acetylated chromatin (Figures 3c, 3d, 4d, 4e and 7b). We speculate that 411 reduced targeting of mutant PBAF $\triangle \mathrm{BD}$ away from the periphery may lead to enhanced

412 heterochromatic spreading decreasing both the size and number of transcriptionally active 413 compartments (Figures 3c and 3d). Our findings are consistent with BAF180's role in 414 promoting H3.3 loading during the formation of functional boundary elements to prevent 415 pericentric heterochromatin spreading in Drosophila ${ }^{42}$. In addition, RSC, the yeast 416 homolog of PBAF, is known to play a major role in establishment of nucleosome free 417 regions at the boundaries of chromatin interaction domains leading to a less compact 418 genome $^{11}$. Therefore PBAF may have conserved role in shaping genome topology via 419 prevention of heterochromatic spreading into compartments containing transcriptionally 420 active genes.

421 Previous studies have focused on the ability of PBAF and related chromatin 422 remodeling complexes to strictly engage facultative heterochromatin and antagonize 423 Polycomb mediated repression ${ }^{43,44}$. Our data indicate that PBAF also functions at the 424 periphery of constitutive HP1 $\alpha$ marked heterochromatic compartments (Figure 4a, right 
425 panel). Reports documented that the BRG1 subunit of PBAF binds to HP1 $\alpha$ and is

426 required for loading of $\mathrm{HP} 1 \alpha$ onto heterochromatin in vitro and in vivo ${ }^{45,46}$. HP1 $\alpha$

427 prevents spreading of $\mathrm{H} 3 \mathrm{~K} 27 \mathrm{me}^{3}$, a well-known target of Polycomb, in pericentric

428 heterochromatin ${ }^{47}$. We speculate that PBAF aids heterochromatic loading of HP1 $\alpha$ at the

429 periphery of constitutive heterochromatic compartments to disrupt spreading of

$430 \mathrm{H} 3 \mathrm{~K} 27 \mathrm{me}^{3}$ and antagonize Polycomb binding. Therefore, our studies uncover a novel

431 role for PBAF in chromatin remodeling of HP1 $\alpha$ marked heterochromatic regions.

432 Different chromatin states can be, in part, defined by their nucleosome stability ${ }^{48}$.

433 Thus, different chromatin states may also be reflected by the genomic residence time of

434 chromatin remodelers in a live cell. Our imaging experiments support this idea by

435 showing that PBAF displayed shorter residence times in H3.3 marked transcriptionally

436 active regions compared to HP1 $\alpha$ containing heterochromatic regions (Figure $5 \mathrm{~d}$ ). This is

437 completely consistent with previous in vitro and in vivo studies showing inherent

438 instability of $\mathrm{H} 3.3$ containing nucleosomes ${ }^{49-51}$. Thus, rapid turnover of PBAF's

439 chromatin occupancy in transcriptionally active regions may directly reflect nucleosome

440 stability (Figure 7b), which could help define different chromatin states.

441 Time-dependent averaging of PBAF chromatin binding trajectories revealed

442 directional movement (Figure 6b). Furthermore, there is a strong correlation between

443 chromatin structure and PBAF's velocity (Figure 6d), with complexes moving slower as

444 nucleosome density increases (Figure 7b). Surprisingly, mutant PBAF lacking BAF180

445 bromodomains moved faster compared to wild-type PBAF in HP1 $\alpha$ marked

446 heterochromatic compartments (Figure 6d). Thus, our data indicate that mutant PBAF

$447 \Delta \mathrm{BD}$ may be mistargeted to distinct local heterochromatin environments containing less 
448 densely packed nucleosomes. This is further supported by data showing that mutant

449 PBAF $\triangle B D$ binds for shorter periods of time relative to wild-type PBAF in HP1 $\alpha$ marked

450 heterochromatic compartments (Figure 5d vs 5e). Alternatively, it is also possible that

451 different subunits and mechanisms, independent of chromatin compaction states, regulate

452 mutant PBAF $\triangle \mathrm{BD}$ 's velocity on heterochromatin.

453 Thus far, numerous research groups have uncovered a plethora of histone post-

454 translational marks (PTM) utilized by chromatin remodelers to regulate transcriptional

455 activation $^{52}$. Previous genomic studies have mapped these histone acetylation marks to

456 broad chromatin domains along with select regions of promoters and enhancers ${ }^{40,41,53}$.

457 Recent studies on 3D organization of the genome revealed compartments containing

458 histone marks associated with activated transcription ${ }^{18,54}$. By characterizing the dynamic

459 genomic binding of PBAF, which recognizes many of these acetylation marks, our work

460 helps to define the spatial and temporal histone modification of chromatin hubs and

461 compartments. Future live-cell single molecule imaging studies of additional histone

462 PTM writers, readers, and the histone marks themselves will shine new light on the

463 spatiotemporal organization of the 4D epigenome. 
Methods

466 Plasmid constructions, biochemistry and generation of cell lines

Details of plasmid construction, biochemical analysis of Halo-BAF180 and

468 generation of cell lines are described in the Supplemental Methods section.

Cells stably expressing Halo-BAF180 WT were grown to a density of $\sim 5 \times 10^{5}$

471 cells on $35 \mathrm{~mm}$ MatTek imaging dishes. Twenty-four hours prior to transfection of Halo-

472 BAF180 $\triangle \mathrm{BD}$, parental U2OS cells were grown to a density of $\sim 1 \times 10^{5}$ cells in $35 \mathrm{~mm}$

473 MatTek imaging dishes. Cells were then transiently transfected with the pFRT-Halo-

474 BAF180 $\triangle \mathrm{BD}$ plasmid and incubated overnight prior to further treatments.

475 Twenty-four hours before labeling, cells were treated with either $2.5 \mu \mathrm{M}$ SAHA

476 (Cayman Chemical) or matching vehicle control (DMSO, 0.25\% final concentration)

477 (Sigma) and were incubated at $37^{\circ} \mathrm{C}$ with $5 \% \mathrm{CO}_{2}$. Immediately prior to imaging, cells

478 were incubated with $0.4 \mathrm{nM} \mathrm{JF549-HTL}$ dye for 15 minutes at $37^{\circ} \mathrm{C}$. Cells were then

479 washed 3 times with 1X PBS, replaced with complete DMEM and incubated for 30

480 minutes at $37^{\circ} \mathrm{C}$ to remove unincorporated dye. Cells were then washed 2 times with $1 \mathrm{X}$

481 PBS and placed in L-15 imaging media (Life technologies) $+10 \%$ FBS for imaging.

482 Dual color live-cell fluorescent labeling of Halo-BAF180 WT/ABD and H3.3-SNAP

483 or SNAP-HP1a

484 Cells stably co-expressing Halo-BAF180 WT along with either H3.3-SNAP or

485 SNAP-HP1 were grown to a density of $\sim 5 \times 10^{5}$ cells on $35 \mathrm{~mm}$ MatTek imaging dishes.

486 Immediately prior to imaging, cells were incubated with $10 \mathrm{nM}$ SNAP-Cell 647-SiR

487 (New England Biolabs) and $0.4 \mathrm{nM}$ JF549-HTL at $37^{\circ} \mathrm{C}$ for a total of 30 and 15 minutes, 
488 respectively. Cells were then washed 3 times with $1 \mathrm{X}$ PBS, replaced with complete

489 DMEM and further incubated for 30 minutes at $37^{\circ} \mathrm{C}$ to remove unincorporated dye.

490 Cells were then washed 2 times with 1X PBS and placed in L-15 imaging media (Life

491 technologies) $+10 \%$ FBS for imaging. Two color labeling of Halo-BAF180 $\triangle \mathrm{BD}$ along

492 with H3.3-SNAP or SNAP-HP1 $\alpha$ was performed as described above, except that Halo-

493 BAF180 $\triangle \mathrm{BD}$ were electroporated with pSNAP plasmids 24-72 hours prior to labeling.

494 Live-cell single molecule imaging of Halo-BAF180 WT/ABD, H3.3-SNAP or SNAP-

495 HP1 $\alpha$

496 All imaging sessions were carried out at room temperature. Cells were 497 continuously illuminated using a $532 \mathrm{~nm}\left(13 \mathrm{~W} / \mathrm{cm}^{2}\right.$, Coherent $)$ or $640 \mathrm{~nm}\left(9.5 \mathrm{~W} / \mathrm{cm}^{2}\right.$, 498 Coherent) laser for JF549-HTL and SNAP-Cell 647-SiR imaging respectively. Time499 lapse two-dimensional (2D) images of single molecules were acquired with a customized 500 inverted Nikon Eclipse Ti microscope with a 100X oil-immersion objective lens (Nikon, 501 1.49 NA) and further magnified 1.5X post-objective. BAF180 images were acquired at 2 $502 \mathrm{~Hz}$ for $\sim 18$ minutes using an EMCCD (iXon, Andor) with a 512 x 512 pixel field of view

503 (final pixel size of $84 \mathrm{~nm}$ ). SNAP imaging proceeded at $2 \mathrm{~Hz}$ for $\sim 4.5$ minutes in cells 504 that also expressed either SNAP-Cell 647-SiR labeled H3.3-SNAP or SNAP-HP1 $\alpha$.

\section{Image Processing and single molecule tracking}

506 Movies of acquired images were processed to subtract background in ImageJ

507 using a rolling ball radius of 50 pixels. Background subtracted movies were subjected to

508 Multi-Target $\underline{\text { Tracking (MTT) to resolve the trajectories of individual molecules }}{ }^{55}$ using

509 a GUI based implementation, SLIMfast ${ }^{56}$. Localization of individual molecules was

510 achieved by fitting Point Spread Functions (PSFs) of discrete single spots with a 2D 
511 gaussian function. Tracking of single molecule chromatin binding events was performed

512 by connecting BAF180 localizations between consecutive frames. Tracking was based

513 upon a maximum expected diffusion constant of $0.05 \mu \mathrm{m}^{2} /$ second and allows for 1.5

514 second gaps in trajectories due to blinking or missed localizations. Track positions from

515 individual trajectories were averaged to generate a 2D projection map of BAF180 binding

516 events over 18 minutes of imaging.

517 Nuclear BAF180 tracks were identified based on the boundaries from 2D

518 projection maps of 180 binding events. BAF180 tracks that fell outside of the nucleus

519 were excluded. Photobleach rates were then determined for each background-subtracted

520 movie based upon exponential decay of the global fluorescence of chromatin bound

521 Halo-BAF180 WT/ABD, H3.3-SNAP, SNAP-HP1 $\alpha$.

\section{Analysis of PBAF chromatin binding residence times}

523 Chromatin binding residence time was determined by plotting a survival curve (1-

524 Cumulative Density Function, 1-CDF) of the track-lengths of chromatin bound Halo-

525 BAF180 in each cell. Single and double-exponential models were then fitted to these 1-

526 CDF plots to determine residence times. One-way ANOVA followed by Tukey's post-

527 hoc t-tests were performed to determine pairwise significance of global residence times

528 and percentages of stable PBAF binding events.

529 Analysis of PBAF clustering in hubs

530 2D projection maps of BAF180 binding events lasting at least one second

531 (Figures $2 \mathrm{e}$ and $2 \mathrm{~g}$ ) or eight seconds (Figure 2c) were expanded 10 fold in the $\mathrm{X}$ and $\mathrm{Y}$

532 directions yielding a final pixel size of $8.4 \mathrm{~nm}$. Areas of high PBAF binding densities

533 were determined by counting the number of binding events within an octagon window 
534 (diameter $168 \mathrm{~nm}$ ) as it was raster scanned across the nucleus of the expanded 2D

535 projection map. Contiguous octagon widows centered on an individual pixel containing at

536 least 3 PBAF binding events were defined and labeled as hubs. The total number of hubs

537 per cell were then normalized to the total PBAF binding events per cell and multiplied to

538 produce the number of PBAF hubs formed per 10,000 PBAF binding events (i.e. Number

539 of PBAF hubs) for each cell. Overall significance was determined with a two-sample

540 Kruskal-Wallis test to determine pair-wise significance.

541 To quantitate PBAF binding latency times in hubs, tracks of binding events in

542 individual hubs were further analyzed to determine the time periods between single

543 PBAF binding events. 1-CDF survival plots of all PBAF latency times in hubs in a single

544 cell were fit with a single, double, and triple exponential decay model. Overall

545 significance was determined with a two-sample Kruskal-Wallis test to determine pair-

546 wise significance.

\section{Mapping of PBAF binding compartments}

548 Contiguous nuclear regions of compartments containing high frequency PBAF

549 binding in cells were determined by raster scanning of a 19 x 19 pixel $\left(2.54 \mu \mathrm{m}^{2}\right)$ window

550 across the nucleus. Binding events that fell within this window were then counted to

551 generate an overall binding density for individual pixels throughout the nucleus. Pixels in

552 the resulting PBAF density map, which displayed a lower binding density than the

553 average global binding density, were filtered out and eliminated. Remaining continguous

554 areas containing at least 10 pixels displaying a high frequency of PBAF binding events

555 were grouped as compartments. For residence time analysis, compartments with fewer

556 than 120 binding events were eliminated to permit more robust statistical analysis of 
557 events. Track lengths for molecules within these remaining compartments were then

558 plotted as a 1-CDF survival curve and fit to single- and double-exponential decay

559 functions. Residence times of the specific binding population for each compartment were

560 plotted as Probability Density Functions (PDF). Statistical differences between genotypes

561 or treatment conditions were then assessed using a two-sample Kolmogorov-Smirnov test.

\section{Characterization of PBAF localization to H3.3 or HP1 $\alpha$ compartments}

563 High binding density H3.3 or HP1 $\alpha$ compartments were mapped using MTT and

564 raster scanning as described above. Remaining compartments were then filtered, such

565 that regions below a threshold of $0.5 \%$ of total nuclear binding events for H3.3 and HP1 $\alpha$

566 were eliminated. PBAF binding events within each compartment were summed and

567 normalized to the total number of PBAF binding events within the cell (nPBIC). This was

568 further normalized to the percentage of the total nuclear area occupied by either H3.3 or

569 HP1 $\alpha$ compartments (nAC). For analysis of binding probability in relation to

570 compartment periphery, the number of PBAF binding events within a 1 pixel $(84 \mathrm{~nm})$

571 band around the perimeter of the compartment was determined and normalized to the

572 total number PBAF binding events in the cell. This was further normalized to the

573 percentage of nuclear area occupied by the 1 pixel $(84 \mathrm{~nm})$ band around the perimeter of

574 the compartment. The outer band of the compartment was then removed and the

575 subsequent binding probability analysis for the 84-168 nm, 169-252 nm, 253-336 nm,

$576 \quad 337-420 \mathrm{~nm}$ bands away from the compartment periphery were performed in a similar

577 manner. Overall significance was determined with a two-sample Kruskal-Wallis test to

578 determine pair-wise significance.

580 Characterization of PBAF binding dynamics within H3.3 or HP1 $\alpha$ compartments

581 Track lengths for PBAF molecules within compartments were plotted as a 1-CDF

582 survival curve and fit to single- and double-exponential decay functions. Residence times 
583 of the specific binding population for each compartment were plotted as Probability

584 Density Functions (PDF). Statistical differences between genotype or treatment

585 conditions were then assessed using a two-sample Kolmogorov-Smirnov test.

586 Velocity analysis of chromatin bound PBAF

587 To assess the average velocity of chromatin bound PBAF within compartments

588 (Figure 6), we analyzed PBAF molecules bound between 7.5 and 15 seconds, based on

589 the average residence times of PBAF within H3.3 and HP1 $\alpha$ marked compartments

590 (Figures 5d and 5e). Localizations (i.e. X, Y positions) from PBAF binding trajectories

591 (Figure 6b) were time averaged using a top hat filter with a sliding window of 5.5

592 seconds. The distance of the displacement between time-averaged localizations from

593 subsequent frames ( 0.5 seconds $)$ was then used to generate a velocity for each individual

594 step throughout the binding trajectory (Figure S9a). Velocities throughout the binding

595 trajectory were then averaged to obtain a mean velocity for an individual PBAF

596 chromatin binding event. Histograms of mean PBAF velocities within compartments

597 were then fit using a four component Gaussian mixture model employing an iterative

598 Expectation Maximization algorithm in MATLAB. The resulting Gaussian mixture

599 models revealed populations containing 4 unique subclasses of velocities for PBAF

600 genomic binding events. The data are represented by the mean percentage and standard

601 deviation of each subclass from 50 independent Gaussian mixture models fit to the

602 histograms of PBAF velocities.

\section{Data Availability}

604 Datasets generated in this study are available at the NIH 4D Nucleome Data portal

605 (data.4dnucleome.org) : 4DNES2DS2N1U, 4DNESL67GMLC, 4DNES3RWS1Y5, 606 4DNES6IJ8J3X, 4DNESC234IRM, 4DNESCE4GQDC, 4DNESGYDFIM4, and 4DNESEAOOIOB.

607 


\section{References}

6091 Lieberman-Aiden, E. et al. Comprehensive mapping of long-range interactions

610 reveals folding principles of the human genome. Science 326, 289-293,

611 doi:10.1126/science.1181369 (2009).

6122 Rao, S. S. et al. A 3D map of the human genome at kilobase resolution reveals

613 principles of chromatin looping. Cell 159, 1665-1680,

614 doi:10.1016/j.cell.2014.11.021 (2014).

6153 Bintu, B. et al. Super-resolution chromatin tracing reveals domains and

616

617

6184 Wang, S. et al. Spatial organization of chromatin domains and compartments in

619

620

621

622

623

624

625

626

627

628

629

630

631

632

633

634

635

636

637

638

639 single chromosomes. Science 353, 598-602, doi:10.1126/science.aaf8084 (2016).

5 Amat, R. et al. Rapid reversible changes in compartments and local chromatin organization revealed by hyperosmotic shock. Genome Res 29, 18-28, doi:10.1101/gr.238527.118 (2019).

6 Stadhouders, R. et al. Transcription factors orchestrate dynamic interplay between genome topology and gene regulation during cell reprogramming. Nat Genet 50, 238-249, doi:10.1038/s41588-017-0030-7 (2018).

7 Heinz, S. et al. Transcription Elongation Can Affect Genome 3D Structure. Cell 174, 1522-1536 e1522, doi:10.1016/j.cell.2018.07.047 (2018).

8 Porter, E. G. et al. PBRM1 Regulates Stress Response in Epithelial Cells. iScience 15, 196-210, doi:10.1016/j.isci.2019.04.027 (2019).

9 Kuzmanov, A., Karina, E. I., Kirienko, N. V. \& Fay, D. S. The conserved PBAF nucleosome-remodeling complex mediates the response to stress in Caenorhabditis elegans. Mol Cell Biol 34, 1121-1135, doi:10.1128/MCB.0150213 (2014).

10 Damelin, M. et al. The genome-wide localization of Rsc9, a component of the RSC chromatin-remodeling complex, changes in response to stress. Mol Cell 9, 563-573 (2002).

11 Hsieh, T. H. et al. Mapping Nucleosome Resolution Chromosome Folding in Yeast by Micro-C. Cell 162, 108-119, doi:10.1016/j.cell.2015.05.048 (2015). Cho, W. K. et al. RNA Polymerase II cluster dynamics predict mRNA output in living cells. Elife 5, doi:10.7554/eLife.13617 (2016). Cisse, II et al. Real-time dynamics of RNA polymerase II clustering in live human cells. Science 341, 664-667, doi:10.1126/science.1239053 (2013).

14 Liu, Z. et al. 3D imaging of Sox2 enhancer clusters in embryonic stem cells. Elife 3, e04236, doi:10.7554/eLife.04236 (2014).

15 Cho, W. K. et al. Mediator and RNA polymerase II clusters associate in transcription-dependent condensates. Science 361, 412-415, doi:10.1126/science.aar4199 (2018). interactions that control gene transcription. Science 361, doi:10.1126/science.aar2555 (2018). gradient. Genes Dev 31, 1784-1794, doi:10.1101/gad.305078.117 (2017). 
65318 Nora, E. P. et al. Spatial partitioning of the regulatory landscape of the X-

654

655

656

657

658

659

660

661

662

663

664

665

666

667

668

669

670

671

672

673

674

675

676

677

678

679

680

681

682

683

684

685

686

687

688

689

690

691

692

693

694

695

696

697

698

inactivation centre. Nature 485, 381-385, doi:10.1038/nature11049 (2012).

19 Mashtalir, N. et al. Modular Organization and Assembly of SWI/SNF Family Chromatin Remodeling Complexes. Cell, doi:10.1016/j.cell.2018.09.032 (2018).

20 Charlop-Powers, Z., Zeng, L., Zhang, Q. \& Zhou, M. M. Structural insights into selective histone $\mathrm{H} 3$ recognition by the human Polybromo bromodomain 2. Cell Res 20, 529-538, doi:10.1038/cr.2010.43 (2010).

21 Slaughter, M. J. et al. PBRM1 bromodomains variably influence nucleosome interactions and cellular function. J Biol Chem 293, 13592-13603, doi:10.1074/jbc.RA118.003381 (2018).

22 Porter, E. G. \& Dykhuizen, E. C. Individual Bromodomains of Polybromo-1 Contribute to Chromatin Association and Tumor Suppression in Clear Cell Renal Carcinoma. J Biol Chem 292, 2601-2610, doi:10.1074/jbc.M116.746875 (2017).

23 Chen, J. et al. Single-molecule dynamics of enhanceosome assembly in embryonic stem cells. Cell 156, 1274-1285, doi:10.1016/j.cell.2014.01.062 (2014).

24 Ricci, M. A., Manzo, C., Garcia-Parajo, M. F., Lakadamyali, M. \& Cosma, M. P. Chromatin fibers are formed by heterogeneous groups of nucleosomes in vivo. Cell 160, 1145-1158, doi:10.1016/j.cell.2015.01.054 (2015).

25 Hassan, A. H., Neely, K. E. \& Workman, J. L. Histone acetyltransferase complexes stabilize swi/snf binding to promoter nucleosomes. Cell 104, 817-827 (2001).

26 Ho, L. \& Crabtree, G. R. Chromatin remodelling during development. Nature 463, 474-484, doi:10.1038/nature08911 (2010).

27 Brownlee, P. M., Chambers, A. L., Oliver, A. W. \& Downs, J. A. Cancer and the bromodomains of BAF180. Biochem Soc Trans 40, 364-369, doi:10.1042/BST20110754 (2012).

28 Filippakopoulos, P. et al. Histone recognition and large-scale structural analysis of the human bromodomain family. Cell 149, 214-231, doi:10.1016/j.cell.2012.02.013 (2012).

29 Kaeser, M. D., Aslanian, A., Dong, M. Q., Yates, J. R., 3rd \& Emerson, B. M. BRD7, a novel PBAF-specific SWI/SNF subunit, is required for target gene activation and repression in embryonic stem cells. J Biol Chem 283, 32254-32263, doi:10.1074/jbc.M806061200 (2008).

30 Kakarougkas, A. et al. Requirement for PBAF in transcriptional repression and repair at DNA breaks in actively transcribed regions of chromatin. Mol Cell 55, 723-732, doi:10.1016/j.molcel.2014.06.028 (2014).

31 Moreira, J. M. \& Holmberg, S. Transcriptional repression of the yeast CHA1 gene requires the chromatin-remodeling complex RSC. EMBO J 18, 2836-2844, doi:10.1093/emboj/18.10.2836 (1999).

32 Van de Vosse, D. W. et al. A role for the nucleoporin Nup170p in chromatin structure and gene silencing. Cell 152, 969-983, doi:10.1016/j.cell.2013.01.049 (2013).

33 Strom, A. R. et al. Phase separation drives heterochromatin domain formation. Nature 547, 241-245, doi:10.1038/nature22989 (2017).

34 Harada, B. T. et al. Stepwise nucleosome translocation by RSC remodeling complexes. Elife 5, doi:10.7554/eLife.10051 (2016). 
69935 Nocetti, N. \& Whitehouse, I. Nucleosome repositioning underlies dynamic gene

700

701

702

703

704

705

706

707

708

709

710

711

712

713

714

715

716

717

718

719

720

721

722

723

724

725

726

727

728

729

730

731

732

733

734

735

736

737

738

739

740

741

742

743

expression. Genes Dev 30, 660-672, doi:10.1101/gad.274910.115 (2016).

36 Vazquez, J., Belmont, A. S. \& Sedat, J. W. Multiple regimes of constrained chromosome motion are regulated in the interphase Drosophila nucleus. Curr Biol 11, 1227-1239 (2001).

37 Chuang, C. H. et al. Long-range directional movement of an interphase chromosome site. Curr Biol 16, 825-831, doi:10.1016/j.cub.2006.03.059 (2006).

38 Clement, C. et al. High-resolution visualization of $\mathrm{H} 3$ variants during replication reveals their controlled recycling. Nat Commun 9, 3181, doi:10.1038/s41467-01805697-1 (2018).

39 Buckley, M. S. \& Lis, J. T. Imaging RNA Polymerase II transcription sites in living cells. Current opinion in genetics \& development 25, 126-130, doi:10.1016/j.gde.2014.01.002 (2014).

40 Bulger, M. Hyperacetylated chromatin domains: lessons from heterochromatin. $J$ Biol Chem 280, 21689-21692, doi:10.1074/jbc.R500004200 (2005).

41 Wang, Z. et al. Combinatorial patterns of histone acetylations and methylations in the human genome. Nat Genet 40, 897-903, doi:10.1038/ng.154 (2008).

42 Nakayama, T., Shimojima, T. \& Hirose, S. The PBAP remodeling complex is required for histone $\mathrm{H} 3.3$ replacement at chromatin boundaries and for boundary functions. Development 139, 4582-4590, doi:10.1242/dev.083246 (2012).

43 Kadoch, C. et al. Dynamics of BAF-Polycomb complex opposition on heterochromatin in normal and oncogenic states. Nat Genet 49, 213-222, doi:10.1038/ng.3734 (2017).

44 Nakayama, R. T. et al. SMARCB1 is required for widespread BAF complexmediated activation of enhancers and bivalent promoters. Nat Genet 49, 16131623, doi:10.1038/ng.3958 (2017).

45 Nielsen, A. L. et al. Selective interaction between the chromatin-remodeling factor BRG1 and the heterochromatin-associated protein HP1alpha. EMBO J 21, 5797-5806 (2002).

46 Lavigne, M. et al. Interaction of HP1 and Brg1/Brm with the globular domain of histone H3 is required for HP1-mediated repression. PLoS Genet 5, e1000769, doi:10.1371/journal.pgen.1000769 (2009).

47 Bosch-Presegue, L. et al. Mammalian HP1 Isoforms Have Specific Roles in Heterochromatin Structure and Organization. Cell Rep 21, 2048-2057, doi:10.1016/j.celrep.2017.10.092 (2017).

48 Deaton, A. M. et al. Enhancer regions show high histone H3.3 turnover that changes during differentiation. Elife 5, doi:10.7554/eLife.15316 (2016).

49 Henikoff, S., Henikoff, J. G., Sakai, A., Loeb, G. B. \& Ahmad, K. Genome-wide profiling of salt fractions maps physical properties of chromatin. Genome Res 19, 460-469, doi:10.1101/gr.087619.108 (2009).

50 Jin, C. \& Felsenfeld, G. Nucleosome stability mediated by histone variants H3.3 and H2A.Z. Genes Dev 21, 1519-1529, doi:10.1101/gad.1547707 (2007).

51 Jin, C. et al. H3.3/H2A.Z double variant-containing nucleosomes mark 'nucleosome-free regions' of active promoters and other regulatory regions. Nat Genet 41, 941-945, doi:10.1038/ng.409 (2009). 
74452 Verdin, E. \& Ott, M. 50 years of protein acetylation: from gene regulation to epigenetics, metabolism and beyond. Nature reviews. Molecular cell biology 16, 258-264, doi:10.1038/nrm3931 (2015).

Roh, T. Y., Cuddapah, S. \& Zhao, K. Active chromatin domains are defined by acetylation islands revealed by genome-wide mapping. Genes \& development 19, 542-552, doi:10.1101/gad.1272505 (2005).

54 Bonev, B. \& Cavalli, G. Organization and function of the 3D genome. Nature reviews. Genetics 17, 772, doi:10.1038/nrg.2016.147 (2016).

\section{Acknowledgements}

We thank Y.J. Chen and C.S. Peng for development of initial Matlab scripts used for

762 advice for analysis of diffusion rates. We thank S. Healton for reagents and technical

763 assistance in acid extraction of histones, and J.C. Wheat for providing SAHA reagent and

764 initial aliquots of acetyl-H3 antibody. We also thank D. Shechter for providing the

765 histone $\mathrm{H} 3$ antibody. This work was supported by a grant from the NIH

766 (8U01DA047729-04, RAC and RHS) as part of the 4D Nucleome project, the NIH

767 (1R01GM126045-01, RAC) and a NIH Medical Scientist Training Program Grant 768 (T32GM007288, CAK).

\section{Author contributions}

771 Designed and supervised experiments: RAC, CAK, SHL, PC, WL, RHS

772 Generated material: CAK (cell lines, constructs), VW (cell lines, constructs), PD (cell

773 lines), LDL (JF dyes)

774 Performed experiments: CAK, SHL, PC, VW 
bioRxiv preprint doi: https://doi.org/10.1101/111674; this version posted August 22, 2019. The copyright holder for this preprint (which was not certified by peer review) is the author/funder. All rights reserved. No reuse allowed without permission.

775 Analyzed data: CAK, SHL, PC, VW, RAC, WL

776 Wrote paper: RAC, WL, CAK

777 Competing Interests

778 The authors declare no competing interests.

779

780 


\section{$781 \quad$ Figure Legends}

782 Figure 1: Spatial analysis of PBAF chromatin binding events using SMT to define

783 binding hubs and compartments.

784 (a) Transcriptionally active genomic compartments (green) contain open chromatin 785 structures associated with acetylated histone marks (e.g. H3K27ac \& H3K14ac) and the

786 H3.3 histone variant. Transcriptionally inactive genomic compartments (red) contain 787 closed chromatin structures associated with select methylated histone marks (e.g. $788 \mathrm{H} 3 \mathrm{~K} 27 \mathrm{me}^{3} \& \mathrm{H} 3 \mathrm{~K} 9 \mathrm{me}^{3}$ ) and heterochromatic protein HP1 $\alpha$. PBAF contains multiple 789 bromodomains within the BAF180 subunit known to bind acetylated histone marks 790 associated with open chromatin compartments.

791 (b) Motion Blur HiLo microscopy (top panel) of a single U2OS cell stably expressing 792 Halo-BAF180 WT. PBAF containing Halo-BAF180 WT molecules that rapidly diffuse in 793 the nucleoplasm are blurred, while chromatin bound PBAF appears as single bright spots

794 (highlighted by red circles, lower panels). Disappearance of a spot (white arrow) is due to 795 unbinding or dissociation of PBAF from the chromatin.

796 (c) Strategy for measuring the non-homogeneous localization of PBAF chromatin binding 797 events in a nucleus. A 2D projection map of PBAF binding events (red dots) in the 798 nucleus over 18 minutes of imaging is shown. A grey box (left panel, zoomed view in the

799 right panel) outlines a representative window of PBAF binding events in a subnuclear 800 region. Binding events occurring within different sized windows $\left(0.064 \mu \mathrm{m}^{2}\right.$ [blue box] 801 and $2.54 \mu^{2}$ [black box], right panel) in this subnuclear region are counted as the 802 window is scanned over the nucleus. PBAF binding density is thereby determined by 
803 counting the number of binding events located within a given size window. Scale bar, 2 $804 \mu \mathrm{m}$.

805 (d) PBAF binding event frequency heat maps were obtained from two different scanning 806 sized windows using the 2D projection map in (c). Regions of high (red) and low (blue)

807 PBAF binding frequency is presented for the cell shown in (c). Top panels are zoomed in

808 views of dashed boxes in lower panels. PBAF hubs (top left panel, white box) were

809 identified as clusters of frequent PBAF chromatin binding to target loci. PBAF hubs were

810 grouped to form distinct compartments (right panels). Scale bar, $0.25 \mu \mathrm{m}$ (top panels) and

$8112 \mu \mathrm{m}$ (bottom panels), respectively.

\section{Figure 2: Spatial and temporal analysis of PBAF binding hubs}

813 (a) A schematic representation where PBAF cycles on and off an array of nucleosomes.

814 (b) Clustering analysis algorithms indicate PBAF binding events (red dots) within hubs

815 (left panel, blue outlines). The right panel displays an expanded inset of a boxed region in

816 the left panel.

817 (c) The number of hubs formed per cell, which express either wild-type PBAF WT or the

818 mutant PBAF $\triangle B D$. N.S. $=$ Not significant, p-value $*=<0.05$ and $* *=<0.01 .(\mathrm{N}=39$

819 WT DMSO cells, $17 \Delta$ BD DMSO cells, 37 WT SAHA cells, and $22 \Delta B D$ SAHA cells)

820 (d) Temporal occupancy of PBAF at a representative chromatin binding hub. Snapshots

821 of PBAF binding foci at different timepoints in movie (seconds) and the appearance of

822 PBAF binding highlighted by white boxes (top panel). The width of the black bars 823 represents the duration of individual PBAF/chromatin binding events in a hub (bottom 
824 panel). White gaps represent latent time periods when the region containing a hub is not

825 occupied by PBAF. Timepoints $(\boldsymbol{\Delta})$ from the top panel where PBAF binds in the hub.

826 Timepoints $(\Delta)$ where PBAF does not bind the hub.

827 (e) Median number of binding events per hub per cell $(\mathrm{N}=39$ WT DMSO cells, $17 \Delta \mathrm{BD}$

828 DMSO cells, 37 WT SAHA cells, and $22 \Delta$ BD SAHA cells). N.S. = Not significant and 829 p-value $*=<0.05$.

830 (f) The long latency time in PBAF binding hubs (N=39 WT DMSO cells, $17 \Delta \mathrm{BD}$

831 DMSO cells, 37 WT SAHA cells, and $22 \Delta$ BD SAHA cells). p-value $* *=<0.01$ and

$832 * * *=<0.001$.

833 For data in panels (c), (e) and (f), the white bar in the solid black box is the median, while

834 the lower and upper boundaries of the black box correspond to the $25^{\text {th }}$ and $75^{\text {th }}$

835 percentiles of the data respectively. Outliers typically represented less than $10 \%$ of the

836 dataset and therefore were omitted for clarity.

837 Figure 3: Spatial analysis of high frequency PBAF binding, actively transcribing 838 H3.3 and heterochromatic HP1 $\alpha$ compartments

839 (a) Filtered heat map indicating regions of high PBAF chromatin binding frequency

840 shown in yellow and red for cells expressing wild-type Halo-BAF180 (i.e. WT, left panel)

841 and Halo-BAF180 $\Delta \mathrm{BD}$ (i.e. $\triangle \mathrm{BD}$, right panel), respectively. Numbers of compartments

842 (yellow boxes) are indicated. The nucleus is outlined by a white dashed line. Scale bar $=$ $8432 \mu \mathrm{m}$. 
844 (b) Filtered heat maps indicating regions of high frequency H3.3 and HP1 $\alpha$ genomic

845 binding shown in yellow and red for cells expressing H3.3-SNAP (left panel) and SNAP-

846 HP1 $\alpha$ (right panel), respectively. Yellow boxes highlight individual compartments. The

847 nucleus is outlined by a white dashed line. Scale bar $=2 \mu \mathrm{m}$.

848 (c) Median number of H3.3 and HP1 $\alpha$ compartments in a cell expressing Halo-BAF180

849 wild-type (WT) or Halo-BAF180 $\Delta \mathrm{BD}(\Delta \mathrm{BD})(\mathrm{N}=32$ WT cells and $\mathrm{N}=31 \Delta \mathrm{BD}$ cells).

850 (d) Median size of H3.3 and HP1 $\alpha$ compartments in a cell expressing Halo-BAF180

851 wild-type (WT) or mutant Halo-BAF180 $\Delta \mathrm{BD}(\Delta \mathrm{BD})(\mathrm{N}=32 \mathrm{WT}$ cells, $31 \Delta \mathrm{BD}$ cells).

852 For data shown in (c) and (d), the white bar in the solid black box is the median, while

853 the lower and upper boundaries of the black box correspond to the $25^{\text {th }}$ and $75^{\text {th }}$

854 percentiles of the data respectively. ( $\mathrm{N}=16$ BAF180 WT/H3.3 cells, 16 BAF180

$855 \Delta \mathrm{BD} / \mathrm{H} 3.3$ cells, $16 \mathrm{BAF} 180 \mathrm{WT} / \mathrm{HP} 1 \alpha$ cells, and $15 \mathrm{BAF} 180 \Delta \mathrm{BD} / \mathrm{HP} 1 \alpha$ cells). N.S. $=$

856 not significant, $\mathrm{p}$ value $* *=<0.01$ and $* * *=<0.001$. Outliers typically represented less

857 than $10 \%$ of the dataset and therefore were omitted for clarity.

858 Figure 4: Spatial analysis of PBAF chromatin binding activities within H3.3 and

859 HP1 $\alpha$ marked compartments

860 (a) Overlap (blue) between areas of high binding frequency compartments for Halo-

861 BAF180 WT (orange) and either H3.3-SNAP (left panel, green) or SNAP-HP1 $\alpha$ (right 862 panel, red). Scale bar, $2 \mu \mathrm{m}$. 
863 (b) Quantification of total area within a cell $\left(\mu \mathrm{m}^{2}\right)$ for PBAF's chromatin binding

864 compartments (orange), transcriptionally active (H3.3, green, top panel) and

865 heterochromatic (HP1 $\alpha$, red, bottom panel) compartments. The percentage of overlaps

866 between compartments is indicated.

867 (c) Distribution of PBAF's total chromatin binding events within transcriptionally active

868 (H3.3, green, top panel) or heterochromatic (HP1 $\alpha$, red, bottom panel) compartments in a

869 cell. The percentage PBAF chromatin binding events within H3.3 or HP1 $\alpha$ marked

870 compartments in a cell is indicated.

871 (d) Representative spatial distribution of PBAF's chromatin binding events (orange dot)

872 within a transcriptionally active H3.3 compartment (left panel) and a HP1 $\alpha$

873 heterochromatic compartment (right panel). The periphery of the H3.3 and HP1 $\alpha$

874 compartment is shown in green and red respectively. The scale bar is indicated.

875

876 (e) Spatial probability of wild-type PBAF (WT, top panels) and mutant PBAF $\triangle B D$

877 binding events (bottom panels) distributed in either H3.3 (left) or HP1 $\alpha$ (right)

878 compartments. $\mathrm{N}=16 \mathrm{WT} / \mathrm{H} 3.3$ cells, $16 \Delta \mathrm{BD} / \mathrm{H} 3.3$ cells, $16 \mathrm{WT} / \mathrm{HP} 1 \alpha$ cells, and 15

$879 \Delta \mathrm{BD} / \mathrm{HP} 1 \alpha$ cells. N.S. $=$ not significant, $\mathrm{p}$ value $*=<0.05, * *=<0.01$ and $* * *=<$

880 0.001. The white bar present in the solid black box is the median, while the lower and

881 upper boundaries of the black box correspond to the $25^{\text {th }}$ and $75^{\text {th }}$ percentiles of the data,

882 respectively. Outliers typically represented less than $10 \%$ of the dataset and therefore

883 were omitted for clarity.

884 Figure 5: Residence time analysis of chromatin-bound PBAF in distinct

\section{5 compartments}


886 (a) Positions of PBAF tracks within transcriptionally active compartments were plotted

887 with residence times (color-coded based on duration) in the third dimension in a 888 representative cell. Compartment (C) identity is listed.

889 (b) 1-Cumulative Distribution Function Plot (1-CDF) of PBAF bound to chromatin in 890 compartment 4 (C4) from the cell presented in (a) was fitted to a single (gray dashed) or 891 two-component (red solid) exponential decay model. Fitting analysis of residence times 892 reveals two populations (i.e. stable and unstable) of PBAF that bound to chromatin.

893 Percentages of the stable and unstable binding populations are listed next to the residence 894 time.

895 (c) Stable residence time of PBAF bound to chromatin for H3.3 marked compartments 18969 (C1 to C9) from the cell presented in (a) is shown.

897 (d) Gaussian fits were generated to show the distribution of stable residence times of 898 chromatin bound wild-type PBAF (WT) within individual H3.3 (green bars, dark green 899 line) and HP1 $\alpha$ (red bars, dark red line) compartments. ( $\mathrm{N}=144$ H3.3 marked 900 compartments from 16 Halo-BAF180 WT cells and N=114 HP1 $\alpha$ marked compartments 901 from 16 Halo-BAF180 WT cells). Bin size $=2$ seconds.

902 (e) Gaussian fits of the distribution of stable residence times of chromatin-bound mutant 903 PBAF BAF180 $\triangle B D$ within individual H3.3 (green bars, dark green line) and HP1 $\alpha$ (red 904 bars, dark red line) compartments. ( $\mathrm{N}=131$ H3.3 marked compartments from 16 Halo905 BAF180 $\Delta$ BD cells, N=141 HP1 $\alpha$ marked compartments from 16 Halo-BAF180 $\Delta$ BD 906 cells). Bin size $=2$ seconds. 


\section{Figure 6: Curvilinear movement analysis of chromatin bound PBAF}

908 (a) Euchromatin contains a low density of nucleosomes resulting in fast local diffusion of

909 chromatin bound PBAF (left panel). Heterochromatin contains densely packed

910 nucleosomes that restrict local diffusion of chromatin bound PBAF (right panel).

911 (b) Representative plots of two nearby PBAF chromatin binding events within similar

912 time frames displaying curvilinear directional non-brownian movement. Localizations

913 from individual PBAF binding trajectories (yellow and blue thin lines) were time-

914 averaged and highlighted (orange and dark blue thick lines). Frame to frame $\mathrm{X}, \mathrm{Y}$

915 displacements were measured from the beginning of the binding event (green dot) to the

916 end (red dot).

917 (c) A representative PDF plot showing PBAF's velocity of individual binding trajectories

918 within a nucleus. $\mathrm{N}=4362$ binding trajectories in 16 PBAF WT cells. Across all

919 conditions, PDF plots were fit with multiple Gaussian models to segment four different

920 populations of distinct velocities (slow, medium, fast1, and fast2). The percentage for

921 each population is listed.

922 (d) Population distribution of velocities for chromatin bound PBAF WT or mutant PBAF

$923 \Delta \mathrm{BD}$ within $\mathrm{H} 3.3$ or HP1 $\alpha$ compartments. The percentage for each population of PBAF's

924 velocity (i.e. fast, medium and slow) within the same compartment is presented. $\mathrm{N}=549$

925 binding trajectories of PBAF WT inside H3.3 compartments from 16 cells. N=527

926 binding trajectories of PBAF WT inside HP1 $\alpha$ compartments from 16 cells. N=613

927 binding trajectories of the mutant PBAF $\triangle \mathrm{BD}$ inside of H3.3 compartments from 16 cells. 
$928 \mathrm{~N}=787$ binding trajectories of the mutant PBAF $\triangle \mathrm{BD}$ inside HP1 $\alpha$ compartments from 15

929 cells.

930 Figure 7: Models of PBAF's dynamic chromatin targeting to shape genome

931 organization.

932 (a) Highly localized histone acetylation in hubs and compartments increases PBAF's

933 association, revisiting and anchored binding via BAF180 bromodomains. Removal of

934 PBAF's bromodomains decreases residence time (elevated $\mathrm{k}_{\text {off }}$ ) in compartments and

935 increases latency between binding events (reduced $\mathrm{k}_{\text {on }}$ ) in hubs.

936 (b) PBAF helps to shape H3.3 compartments by binding to boundary elements at the

937 periphery to prevent invasion of heterochromatin into transcriptionally active

938 compartments. In H3.3 rich euchromatic compartments, less stable chromatin results in

939 PBAF binding for a shorter duration of time than within stable HP1 $\alpha$ rich

940 heterochromatin. PBAF's residence time on chromatin is proportional to chromatin

941 stability. The velocity of genomic bound PBAF is decreased in densely packed

942 heterochromatin relative to H3.3 rich chromatin. Therefore, PBAF's velocity may reflect

943 chromatin compaction surrounding target nucleosomes. 
Figure 1

a

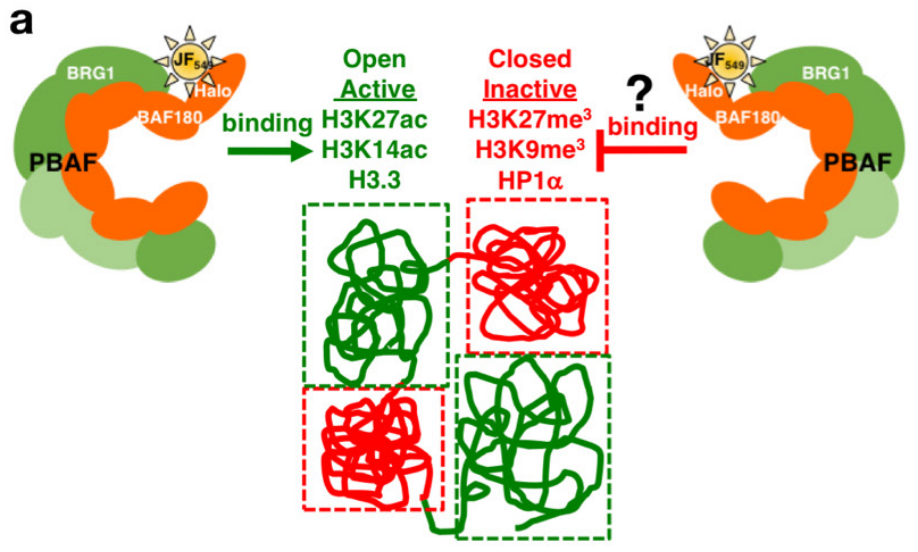

Kenworthy_Fig. 1

b
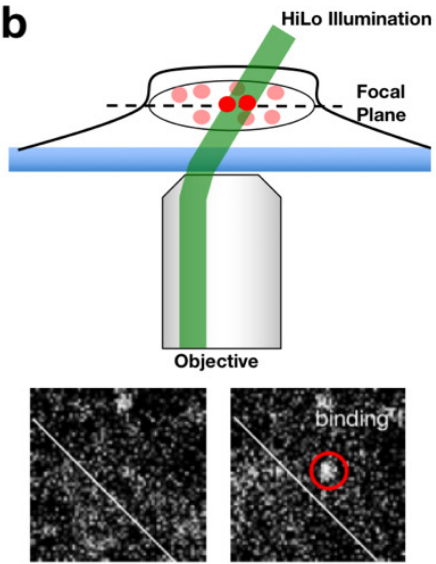

$-0.5 \mathrm{~s}$

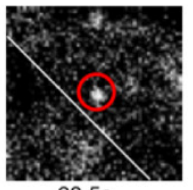

$28.5 \mathrm{~s}$

C

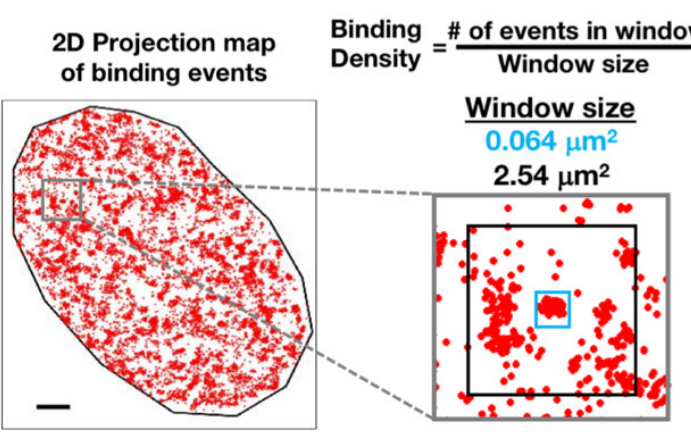

$\sim 1.7 \mu$ m diameter

Chromatin type d

$\sim 0.25 \mu \mathrm{m}$ diameter $\sim 10-20 \mathrm{Kbp}$

Target loci

$0.064 \mu \mathrm{m}^{2}$ window
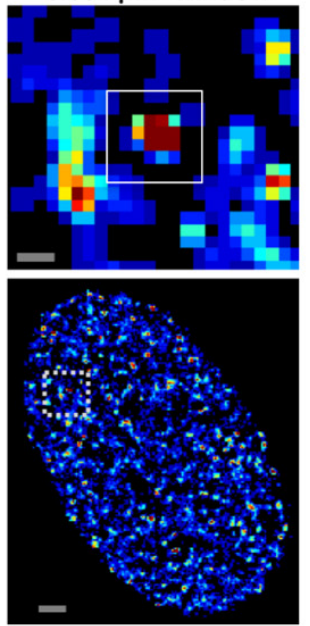

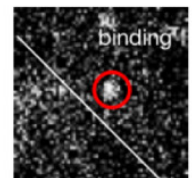

Os

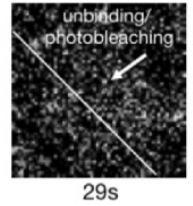

29s

Compartment $\sim \mathrm{Mbp}$ $2.54 \mu \mathrm{m}^{2}$ window

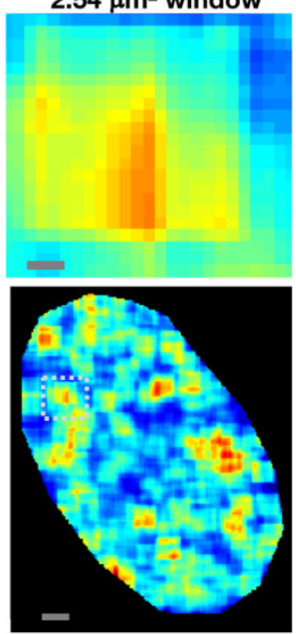


Figure 2

Kenworthy_Fig. 2

a $1^{\text {st }}$ Binding Event on Array

$2^{\text {nd }}$ Binding Event on Array

$3^{\text {rd }}$ Binding Event on Array

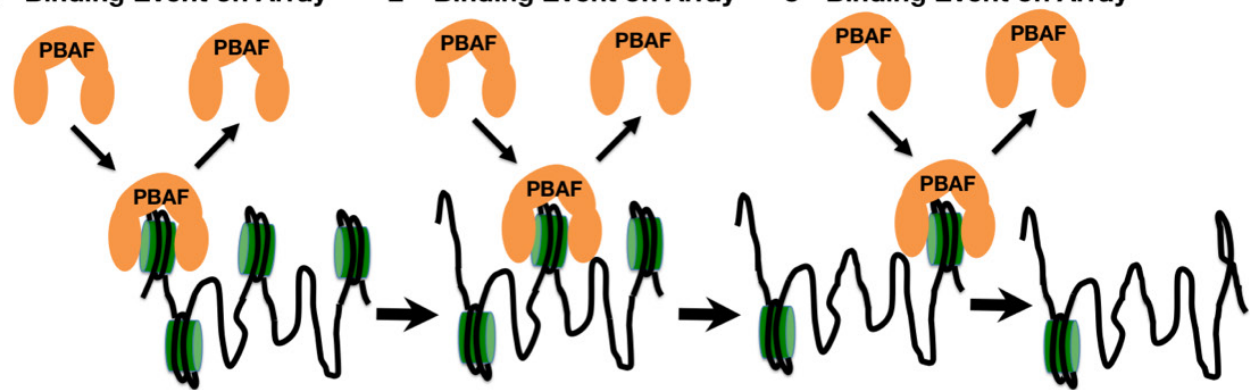

b

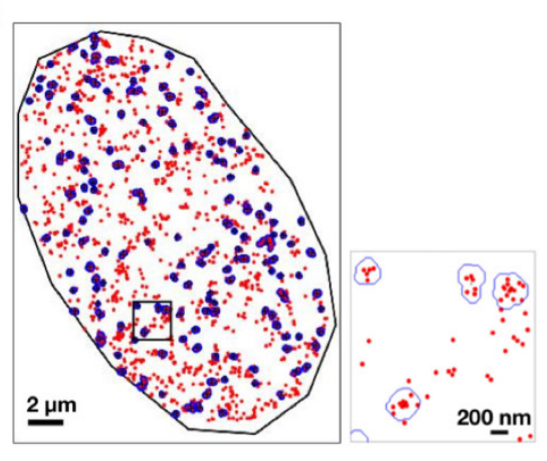

C

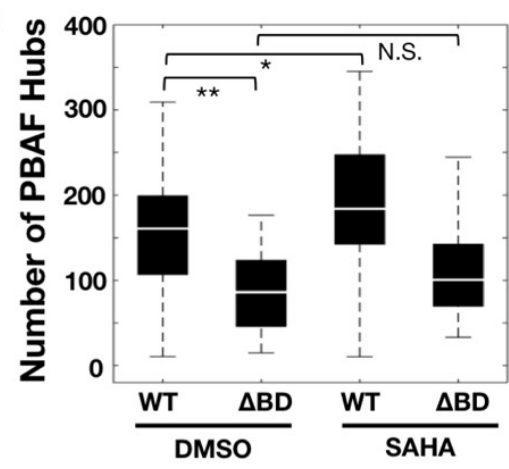

d

58 s 193 s 320 s $475 s 525$ s 673 s 750 s 820 s
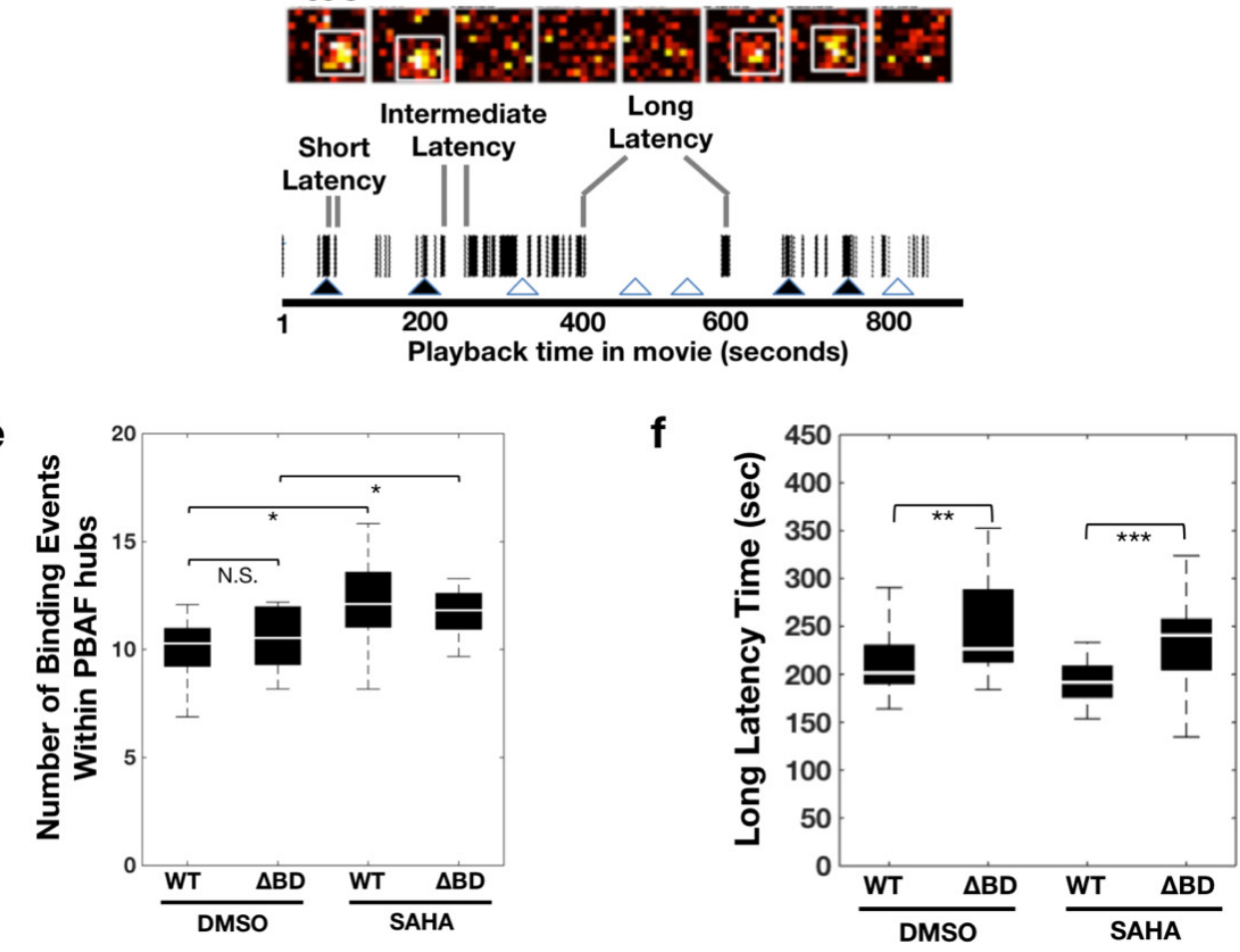
Figure 3

Kenworthy_Fig. 3

a

Filtered PBAF binding frequency map
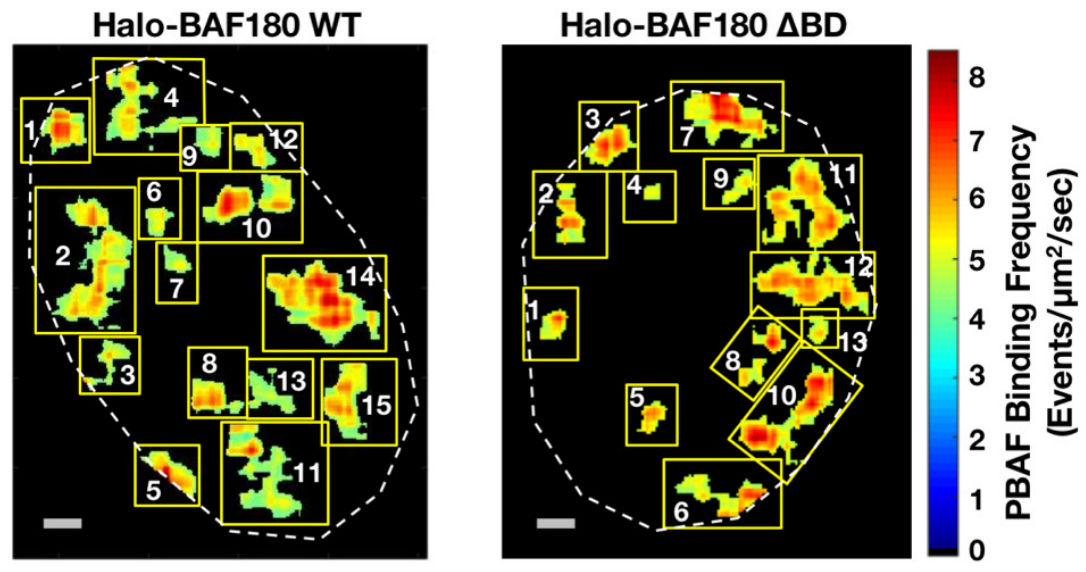

b
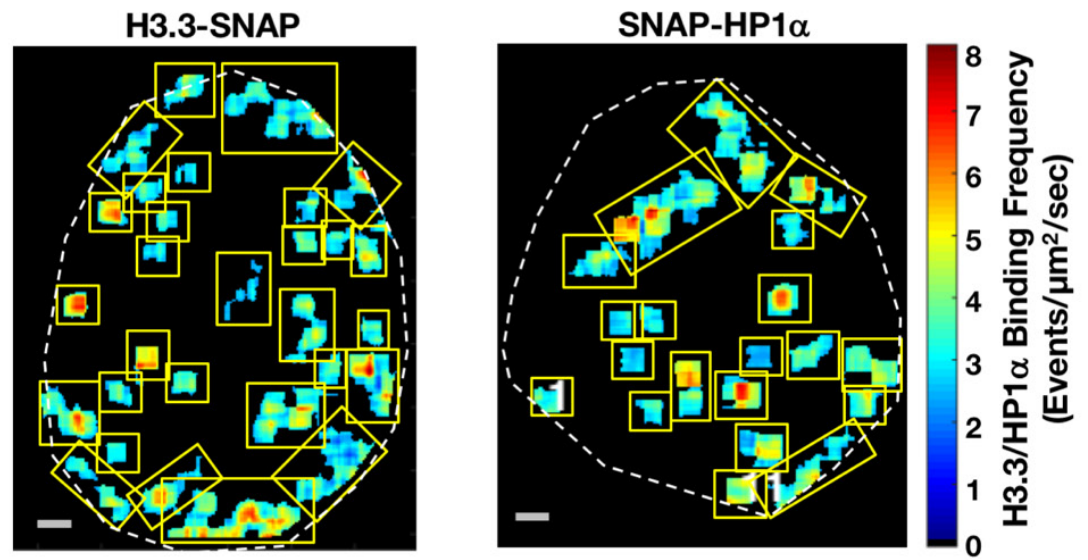

C

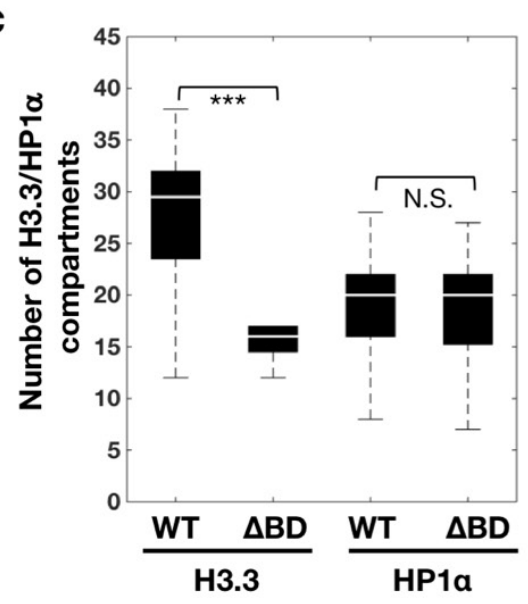

d

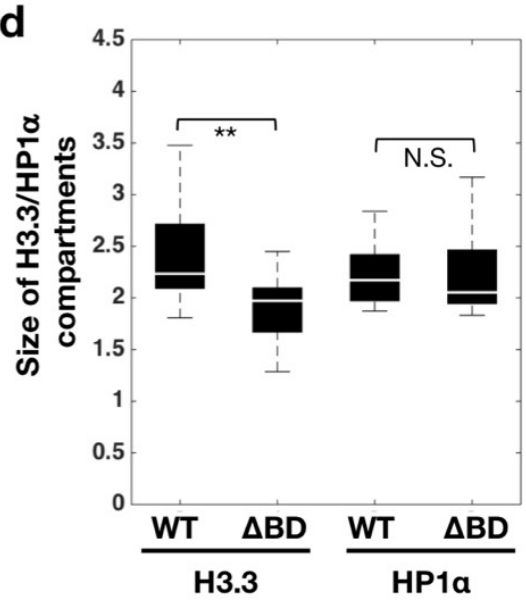


Figure 4

Kenworthy_Fig. 4
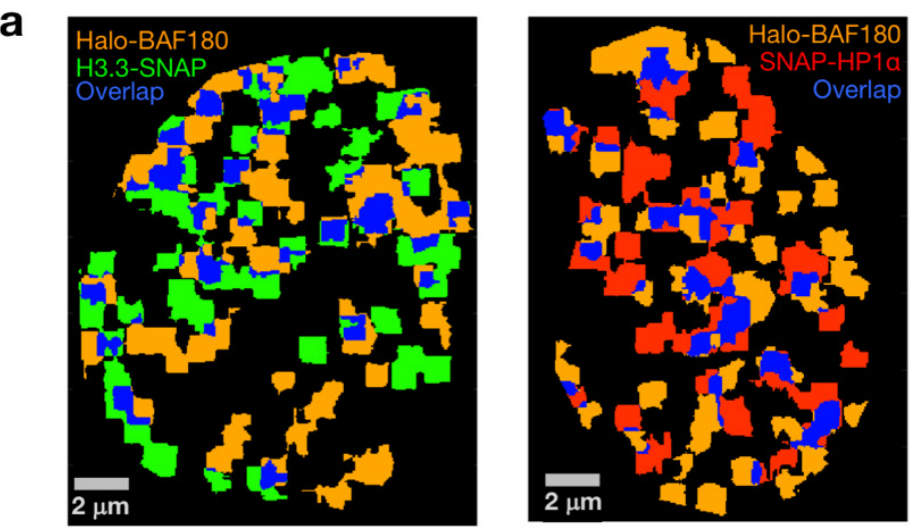

b Total Compartment Area PBAF (27\% overlap)

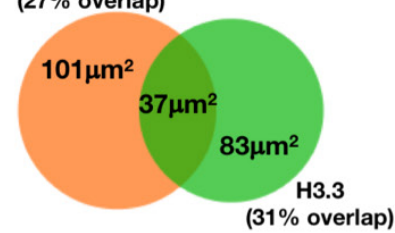

PBAF

(24\% overlap)

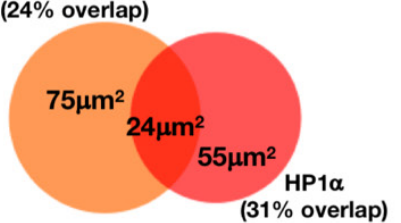

C Total Chromatin Binding Events

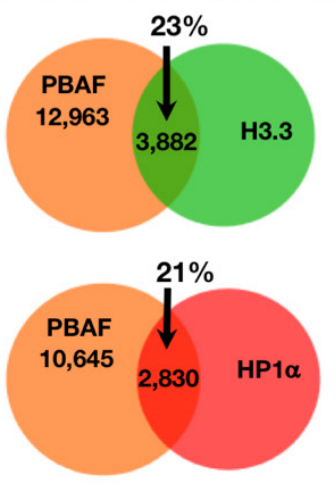

d

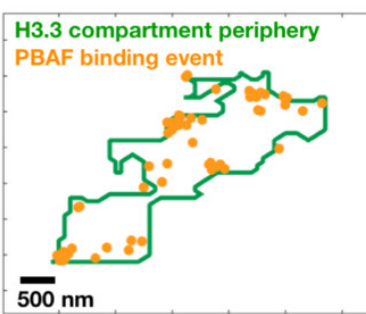

e PBAF WT

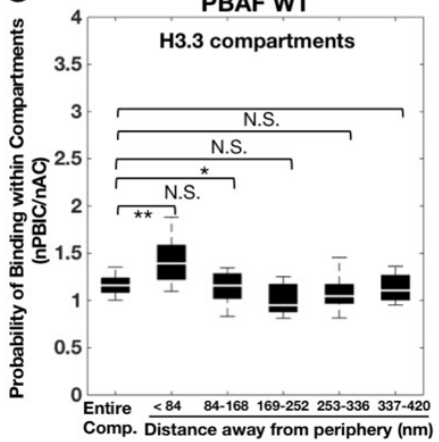

PBAF $\triangle B D$

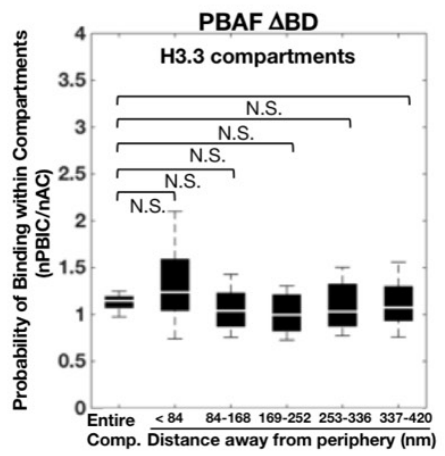

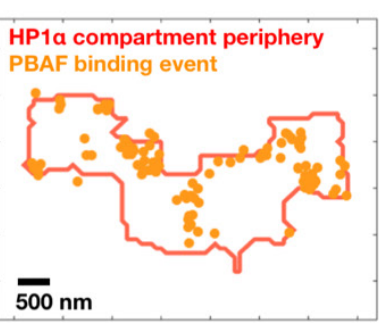

$\overline{500} \mathrm{~nm}$

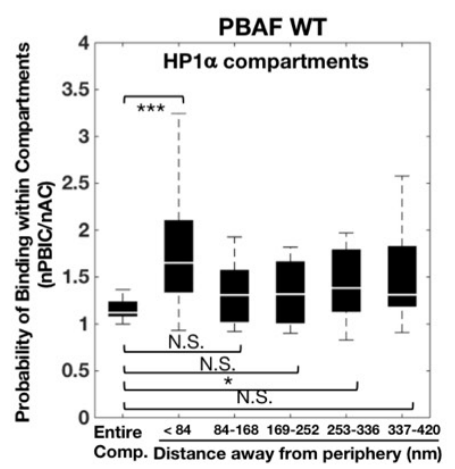

PBAF $\triangle B D$

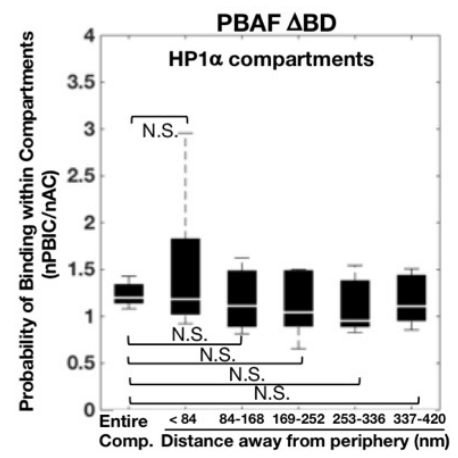


Figure 5

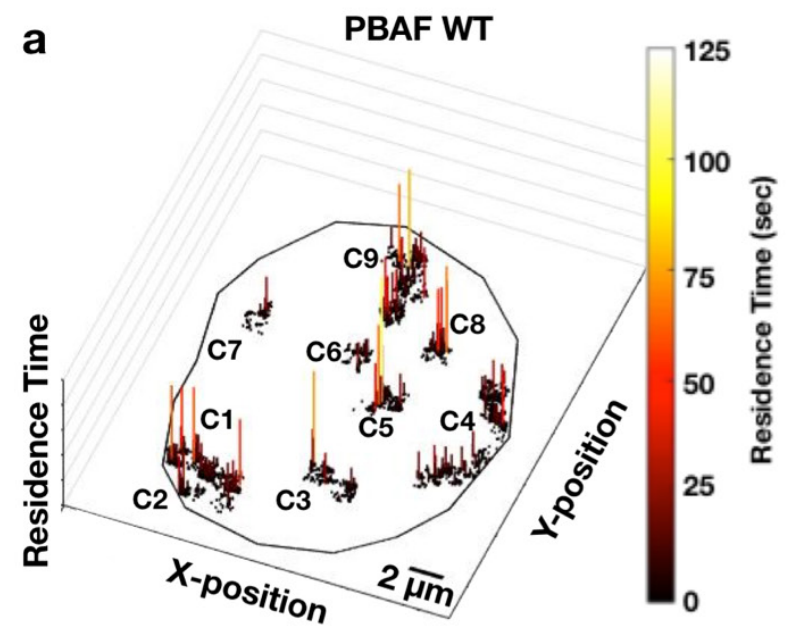

Kenworthy_Fig. 5

b

PBAF's residence time in $\mathrm{H} 3.3 \mathrm{C} 4$

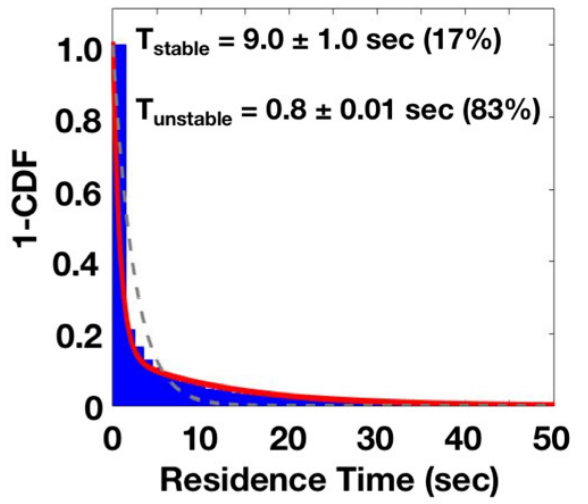

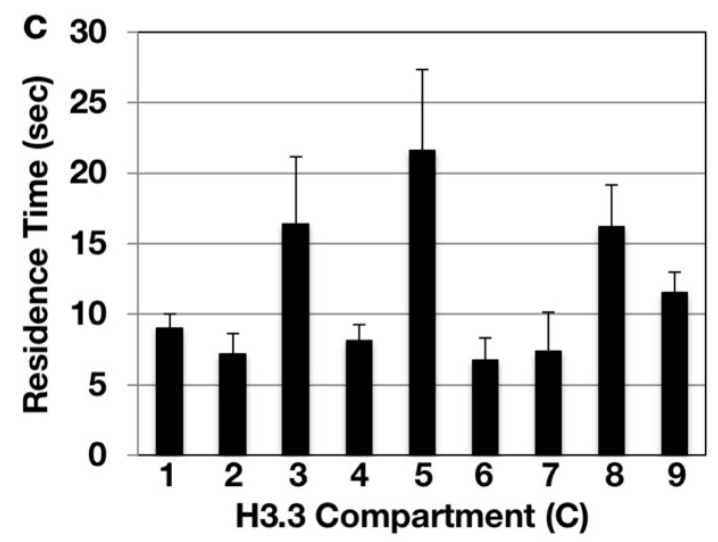
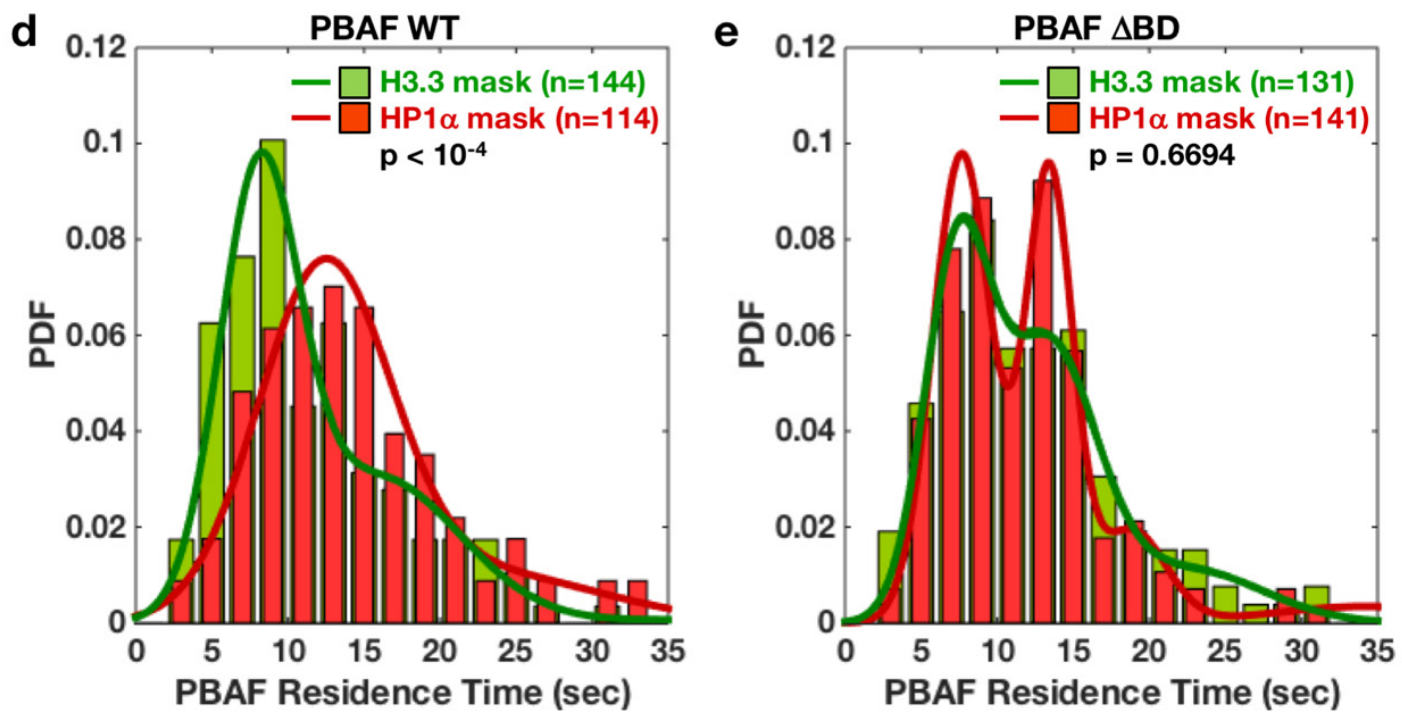
Figure 6

a
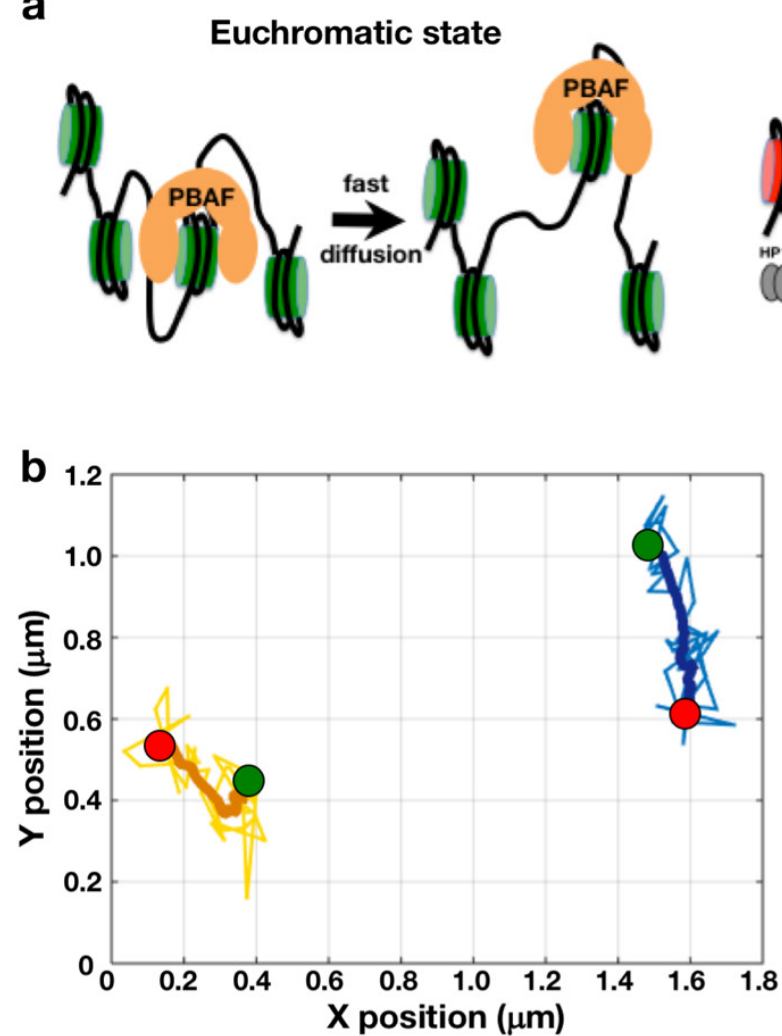

Kenworthy Fig. 6
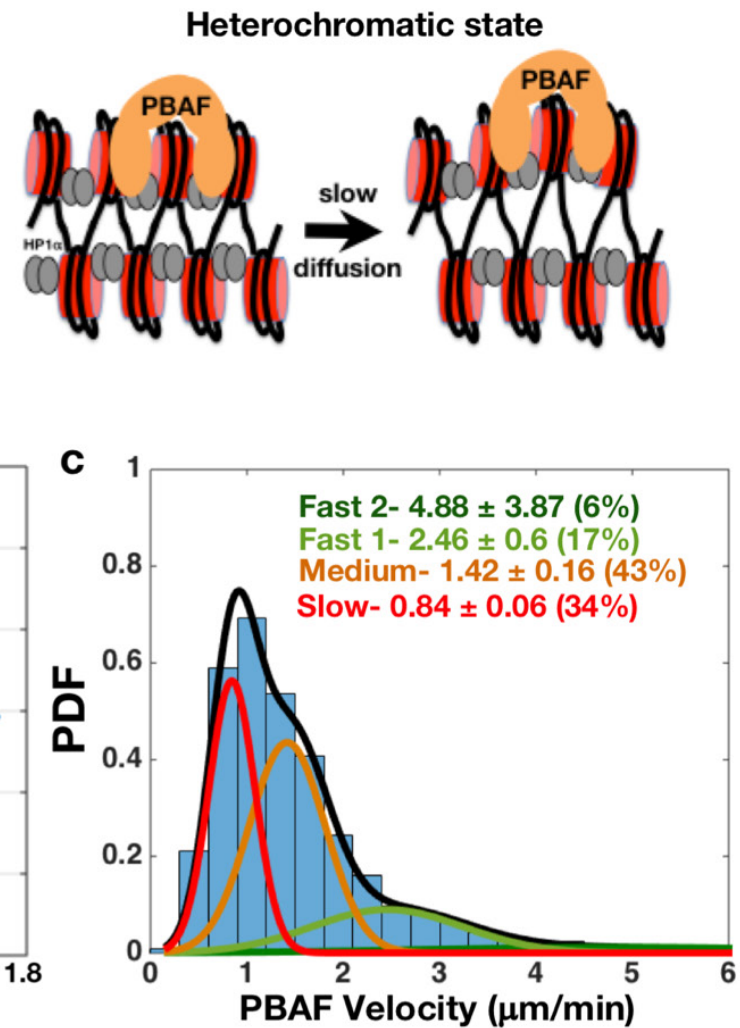

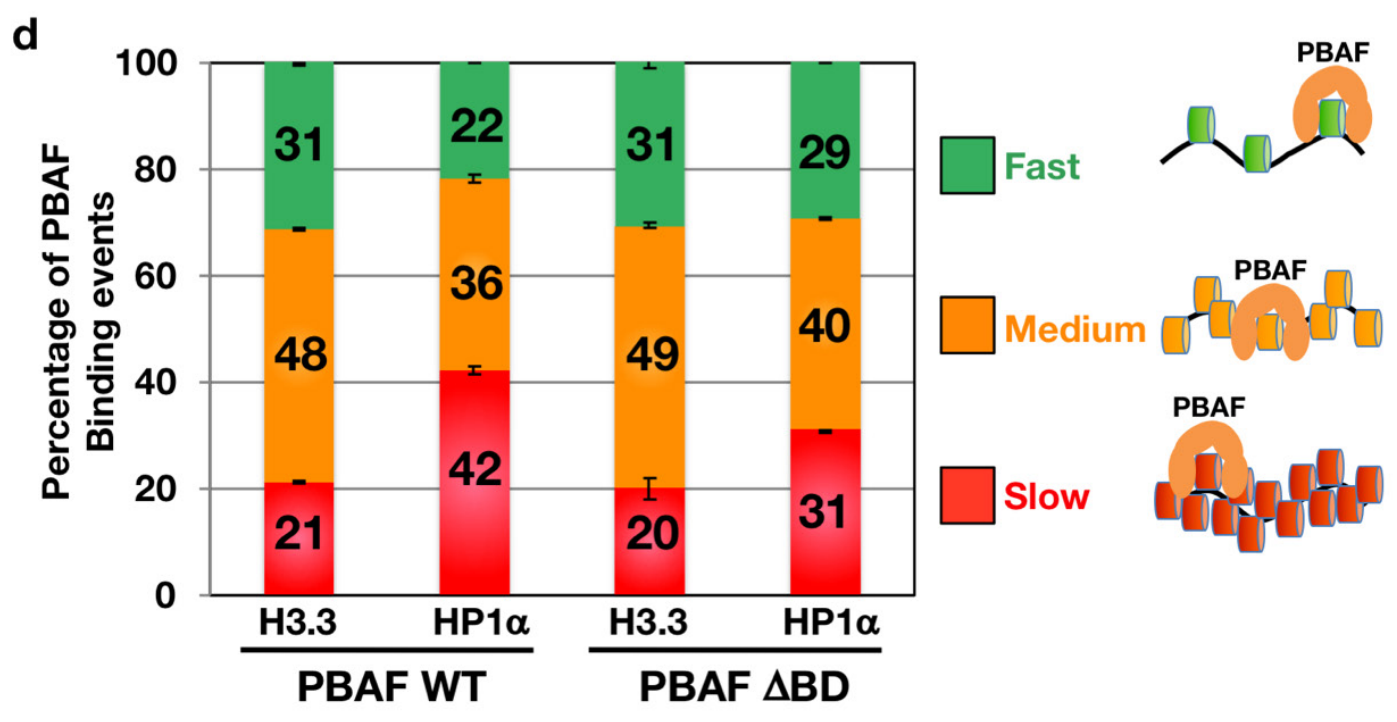

950 
Figure 7

Kenworthy_Fig. 7
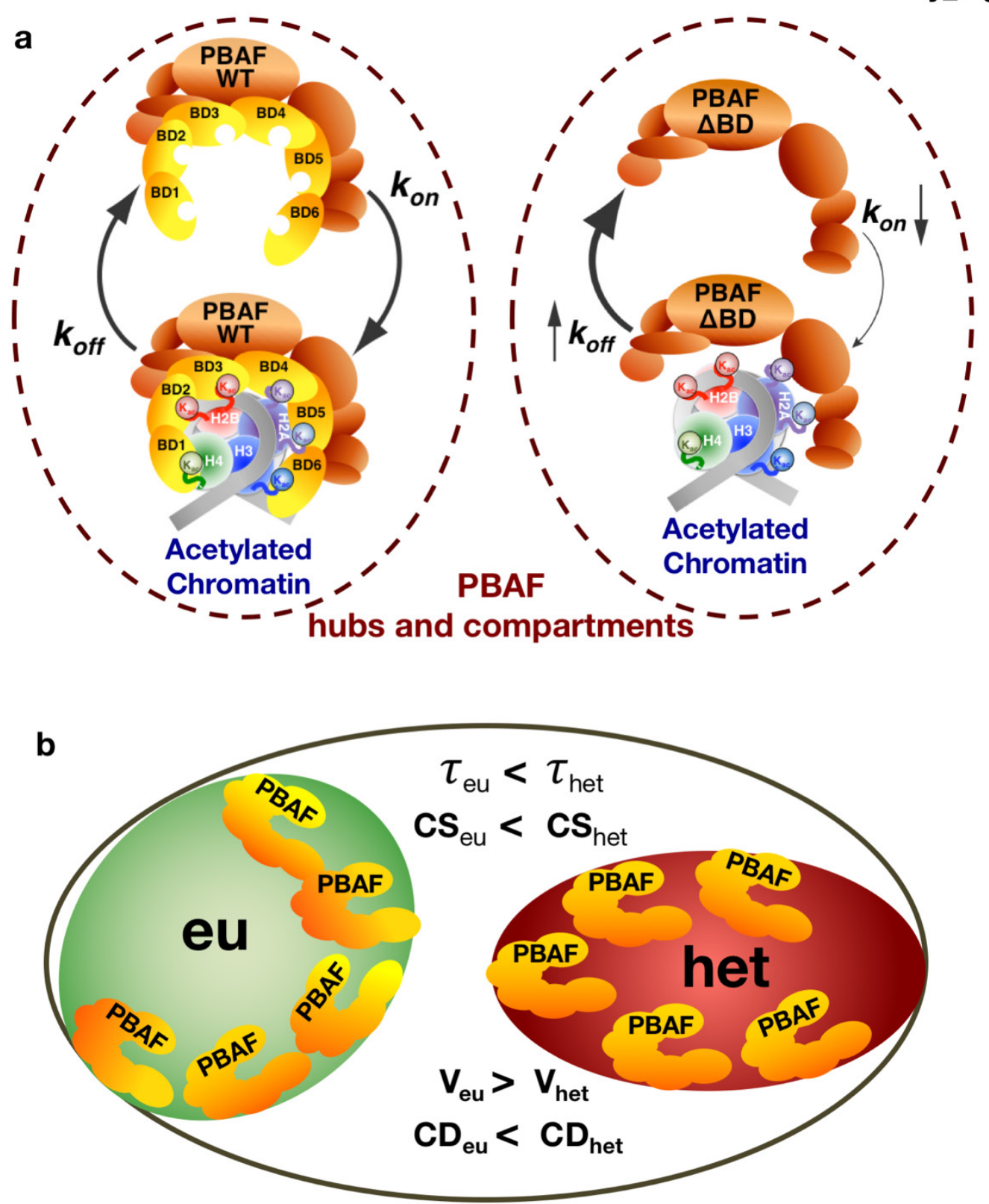

eu H3.3 Euchromatin het HP1a Heterochromatin

PBAF residence time $(\mathcal{T}) \propto$ Chromatin stability (CS) PBAF Velocity $(V) \propto$ Chromatin density (CD) 